\title{
$\alpha$-Dystroglycan is essential for the induction of Egr3, a transcription factor important in muscle spindle formation
}

\author{
by \\ Stacey Catherine Williams \\ A thesis \\ presented to the University of Waterloo \\ in fulfillment of the \\ thesis requirement for the degree of \\ Master of Science \\ in \\ Biology
}

Waterloo, Ontario, Canada, 2009

(C) Stacey Catherine Williams 2009 


\section{Author's declaration}

I herby declare that I am the sole author of this thesis. This is a true copy of the thesis, including any required final revisions, as accepted by my examiners.

I understand that my thesis may be made electronically available to the public. 


\section{Abstract}

Muscle spindle fibers are specialized stretch receptors that allow the perception and coordination of limb movement. Differentiation of muscle spindles is initiated by signals derived from the in growing Ia sensory neurons during development. The sensory neuron secretes neuregulin which binds and signals through the ErbB receptors to initiate a signaling cascade. This cascade results in the expression of a specific repertoire of genes, one of which is the transcription factor Egr3, which is necessary in the development of muscle spindles.

Signaling occurs efficiently when the postsynaptic receptors are clustered into large aggregates in apposition to an innervating nerve. Using what is known about acetylcholine receptor clustering at neuromuscular junctions as a model, this study shows the importance of the basal lamina proteins agrin and laminin and their shared receptor $\alpha$ dystroglycan in aggregating ErbB receptors at sensory synapses. The study also shows that signaling through these receptors subsequently results in increased expression of Egr3, the transcription factor critical to muscle spindle fiber differentiation. Using an $\alpha-$ dystroglycan silenced culture, it is shown that $\alpha$-dystroglycan is necessary to induce neuregulin, laminin and agrin induced Egr3. In these same myotube cultures there is also a reduced number of $\mathrm{AChR}-\mathrm{ErbB} 3$ colocalized aggregates and this is not rescued with the addition of laminin. Taken together, these results suggest an essential role for basal lamina components and $\alpha$-dystroglycan, molecules that are crucial in acetylcholine receptor aggregation at neuromuscular junctions, in the induction of the transcription factor Egr3, a critical transcription factor involved in muscle spindle fiber differentiation. 


\section{Acknowledgements}

Firstly, I am extremely thankful to my supervisor, Dr. Christian Jacobson, for all of his guidance, knowledge, and understanding. I appreciate all of the support and knowledge he has passed on to me throughout completing this degree, both personally and professional. His advice was always appreciated.

I am also grateful to my committee members Dr. Bernard Glick and Dr. Bruce Reed. Their input and guidance is greatly appreciated.

I would also like to thank Dr. Mungo Marsden for all of his advice and assistance in the lab and his willingness to always make the time to answer my questions and help me out. Your experience and patience really allowed be to move forward in my experiments and feel confident in my abilities as a researcher. Thank you also for the use of lab equipment and microscopes.

Thank you to all of my friends and fellow grad students. Thank you to Malwina Mencel and Michelle Nash for always being a joy to work with and a source of support. Also a big thank you to Colleen Ryan and Jen Czarny, your research expertise and advice has come in handy on numerous occasions and your ability to always lightening the mood was much appreciated.

I am grateful to my husband Tom Straus for all his love, support and patients. Your ability to make me see problems as challenges and put everything into perspective has been monumental in my success. Thank you to my parents Denise and Chris Williams, for all of your love and support and the constant encouragement and unconditional support of my academic pursuits. Thank you so much.

Finally, I would like to thank the University of Waterloo for this opportunity and financial support. This research was funded by the University of Waterloo and NSERC grants. I would also like to thank OGS for the graduate scholarship funding. 


\section{Table of Contents}

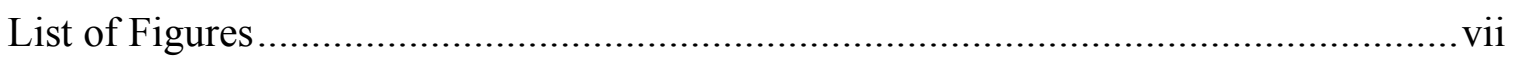

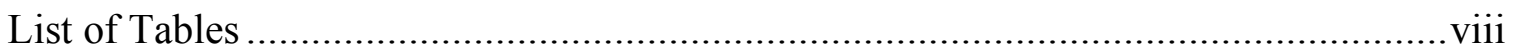

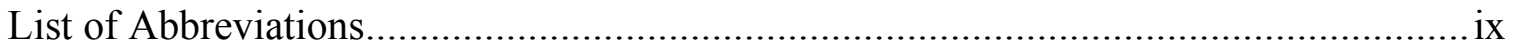

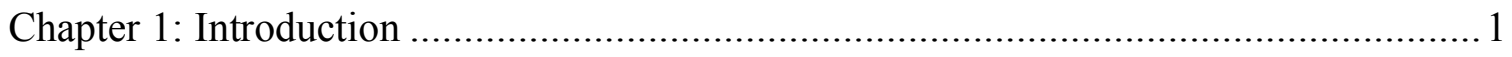

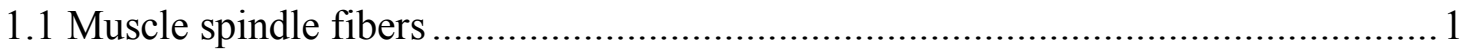

1.2 Development of muscle spindle fibers.............................................................. 3

1.3 NRG signaling is essential for muscle spindle fiber differentiation......................... 3

1.4 AGRN signaling results in aggregation of synapse-specific proteins .....................5

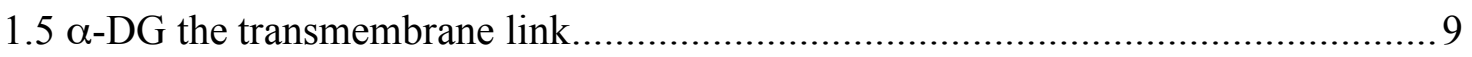

1.6 LN a condenser and stabilizer of AChR synaptic clusters .................................... 15

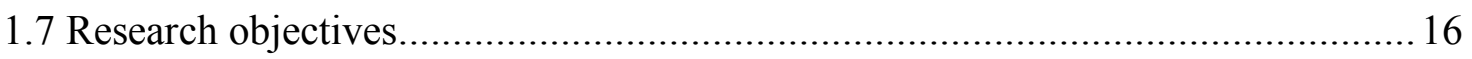

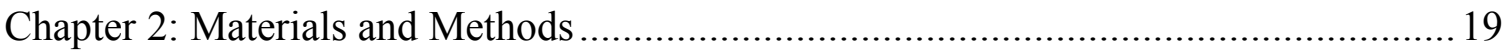

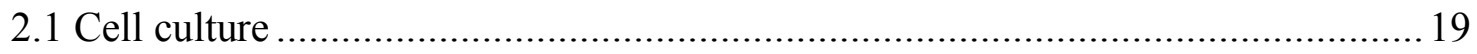

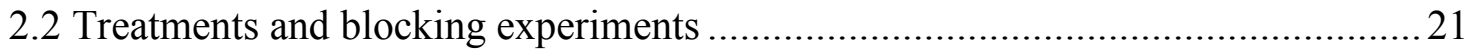

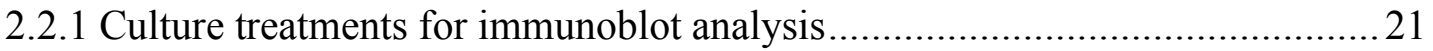

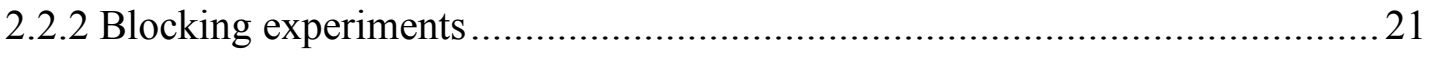

2.2.2 Culture treatments for immunofluorescence..............................................22

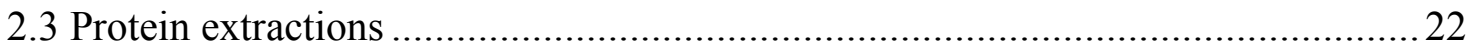

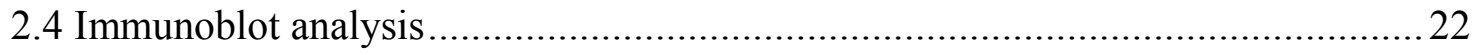

2.5 Immunofluorescence 
Chapter 3: Results

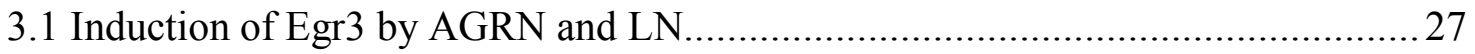

$3.2 \alpha-\mathrm{DG}$ is necessary for NRG, ARGN and LN induced Egr3 expression................ 36

3.3 LN-stimulated Egr3 induction is reduced by NRG and ErbB inhibitors .............. 49



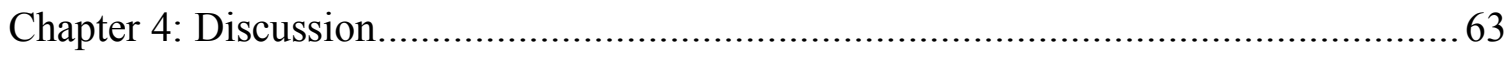

4.1 AGRN and LN can induce Erg3 independently from NRG............................63

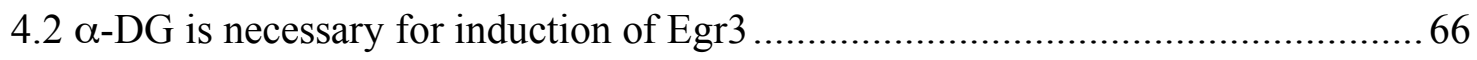

$4.3 \alpha$-DG deficiency reduces the number of AChR and ErbB3 clusters...................69

4.4 Conclusions ......................................................................................... 70

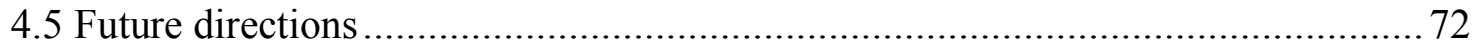

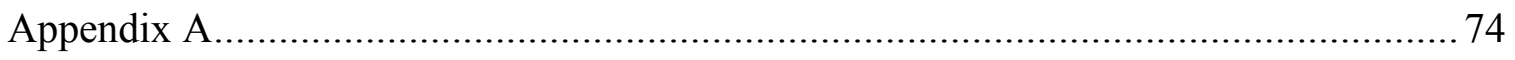

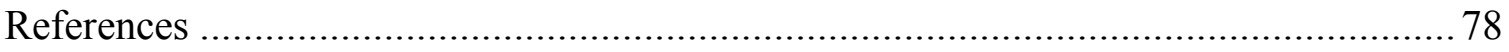




\section{List of Figures}

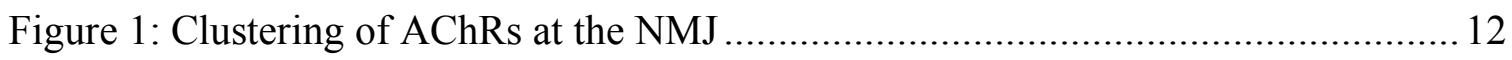

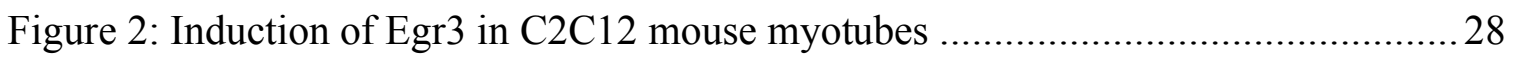

Figure 3: NRG and AGRN induction of Egr3 over 24 hours.................................... 31

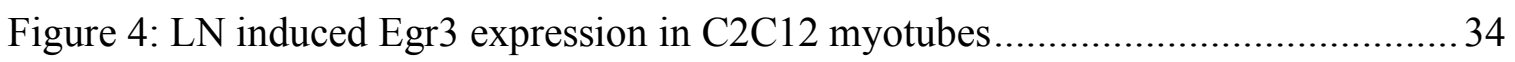

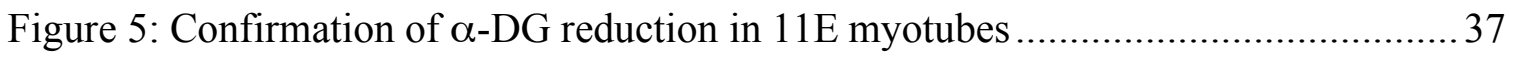

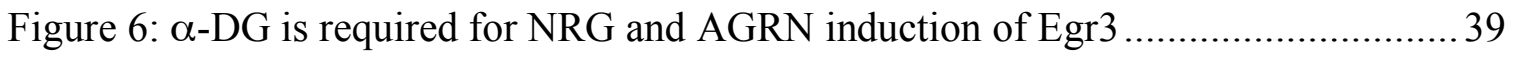

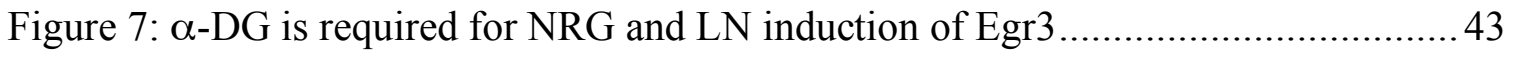

Figure 8: Blocking $\alpha-D G$ reduced the induction of Egr3 .................................... 45

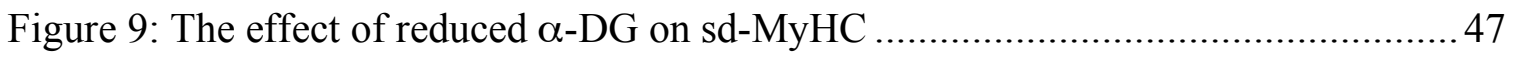

Figure 10: Blocking NRG reduces LN-induced Egr3 expression ............................51

Figure 11: Blocking ErbB receptor signaling reduces LN-induced Egr3 expression.......53

Figure 12: Reduced $\alpha$-DG decreases the number of AChR and ErbB3 aggregates .........57

Figure A1: Western blot of sd-MyHC hybridoma medium ................................... 74 


\section{List of Tables}

Table 1: Overlap of ErbB3 clusters with AChR aggregates..................................... 61

Table A1: AChR/ErbB3 cluster counts and calculations ........................................ 76 


\section{List of Abbreviations}

\begin{tabular}{|c|c|}
\hline$\alpha$-BTX & alpha-bungarotoxin \\
\hline$\alpha-\mathrm{DG}$ & alpha-dystroglycan \\
\hline$\beta-\mathrm{DG}$ & beta-dystroglycan \\
\hline $\mathrm{AChE}$ & Acetylcholine esterase \\
\hline AChR & Acetylcholine \\
\hline AGRN & Agrin \\
\hline ATCC & American Type Culture Collection \\
\hline ATP & Adenosine triphosphate \\
\hline CNS & Central nervous system \\
\hline DGC & Dystrophin-associated glycoprotein complex \\
\hline DMEM & Dulbecco’s Modified Eagle's Medium \\
\hline Dok-7 & Docking protein 7 \\
\hline D-PBS & Dulbecco's Phosphate-buffered Saline \\
\hline DRG & Dorsal root ganglion \\
\hline $\mathrm{E}(\#)$ & Embryonic day \# \\
\hline $\mathrm{ECM}$ & Extracellular matrix \\
\hline EGF & Epidermal growth factor \\
\hline Egr3 & Early growth response factor 3 \\
\hline FBS & Fetal bovine serum \\
\hline GTPases & Guanidine triphosphatases \\
\hline HRP & Horseradish peroxidase \\
\hline
\end{tabular}




$\begin{array}{ll}\text { Ig } & \text { Immunoglobulin } \\ \text { LG } & \text { Laminin-like G-protein } \\ \text { LN } & \text { Laminin } \\ \text { Lrp4 } & \text { Lipoprotein receptor } 4 \\ \text { MuSK } & \text { Muscle specific kinase } \\ \text { NMJ } & \text { Neuromuscular Junction } \\ \text { NT-3 } & \text { Neurotrophin-3 } \\ \text { NRG } & \text { Neuregulin } \\ \text { PBS } & \text { Phosphate-buffered saline } \\ \text { PI3K } & \text { Phosphatidylinositol-3'-kinase } \\ \text { SD } & \text { Standard Deviation } \\ \text { Sd-MyHC } & \text { Slow-developmental myosin heavy chain } \\ \text { SDS-PAGE } & \text { Sodium dodecyl sulfate polyacrylamide gel electrophoresis } \\ \text { Tid1 } & \text { Tumorous imaginal Discs 1 } \\ \text { TrkC } & \text { Tyrosine kinase C }\end{array}$




\section{Chapter 1}

\section{Introduction}

Muscle spindle fibers are specialized muscle stretch receptors. Formed as a result of reciprocal signaling between the muscle and nerve, neuregulin (NRG) is believed to be the required signal provided by the approaching Ia sensory neuron during development that initiates the differentiation of muscle fibers into spindles (Hippenmeyer et al., 2002). The direct molecular signaling mechanisms between the sensory neurons and developing muscle involved in this process have been well documented in past studies, but the roles of muscle basal lamina components such as agrin (AGRN) and laminin (LN) and their common receptor in skeletal muscle, $\alpha$-dystroglycan ( $\alpha$-DG), on this process have not previously been described.

\subsection{Muscle spindle fibers}

Proprioception is the sensation of axial body position and the awareness of limb and body movement through space. Muscle spindle fibers, also referred to as mechanoreceptors, are the specialized sensorimotor organs that permit proprioceptive communication from the periphery to the central nervous system (CNS). Proprioceptive deficits or the inability to sense limb and body movement and position results in difficulties carrying out basic tasks that require coordinated body and limb movement, such as walking. Muscle spindle fibers are also involved in sensorimotor behaviors such as balance and posture (Stapley et al., 2002). Age-associated morphology changes in muscle spindle fibers observed in the elderly may result in decreases in proprioception, 
which could contribute to the increases in ataxia and falls amongst senior populations (Swash and Fox, 1972); (Kararizou et al., 2005). Similar, but more severe abnormalities in gait, as well as scoliosis and resting tremors, are observed in mice deficient for the early growth response three $(\operatorname{Egr} 3)$ transcription factor. In Egr3 null animals there is a complete lack of muscle spindle fiber differentiation (Tourtellotte and Milbrandt, 1998).

The specialized structure of muscle spindle fibers allows them to carry out their important and specialized function. They are small, encapsulated sensory organs that lie in parallel with skeletal (extrafusal or contractile) muscle fibers. Muscle spindles consist of several highly specialized intrafusal muscle fibers, which contain motor neuron endings near the outer ends and the sensory endings within the equatorial region (Hunt, 1990; Zelena, 1994) and a number of extrafusal fibers. The intrafusal muscle fibers can be described as either bag fibers or nuclear chain fibers. Sensory innervation of these fibers by Ia afferents is distinct and characterized by a pattern of extensive annular spirals (Hunt, 1990). Secondary sensory endings are also present around nuclear chain and bag ${ }^{2}$, and are smaller group II sensory axons (Hunt, 1990). Motor innervation is supplied by small $\gamma$-motor neurons, which innervate the extrafusal muscle fibers in the spindle fiber. These motor endings can be distinguished as dynamic or static (Hunt, 1990). The dynamic axons increase the dynamic response of the motor endings when the length of the muscle is changing, while the static axons act to stabilize at a steady length (Hunt, 1990). This is how muscle spindles signal changes in muscle length to the CNS, which allows the perception of body position and control of muscle contraction (Hunt, 1990). This is why degradation or complete lack of muscle spindles fibers results in gait ataxia and other abnormalities in sensorimotor behaviors (Tourtellotte and Milbrandt, 1998). 


\subsection{Development of muscle spindle fibers}

In rodents the development of muscle spindles has been well researched. Differentiation is initiated early in embryonic development and nascent muscle spindle fibers initially appear by embryonic day seventeen [E(17); (Zelena, 1994)], and spindles continue to mature well into postnatal life (Kucera et al., 1988); (Zelena, 1994), Early studies revealed that it is the Ia afferent (sensory) axon that provides the necessary signal input to initiate muscle spindle differentiation, as surgical elimination of sensory, but not motor neurons, results in the rapid deterioration of spindle fibers (Kucera and Walro, 1992a; Kucera and Walro, 1992b; Kucera et al., 1993). Further research showed that mice with mutations that impair the function of neurotrophin-3 (NT-3) or the NT-3 receptor, tyrosine kinase $\mathrm{C}(\operatorname{TrkC})$, fail to develop spindle fibers (Ernfors et al., 1994; Klein et al., 1994). NT-3 is a survival factor provided by fetal muscle and is the ligand of $\operatorname{TrkC}$, which is expressed on proprioceptive sensory neurons. Elimination of either of these factors results in the absence of proprioceptive sensory neurons, and thus, the lack of muscle spindles (Ernfors et al., 1994; Klein et al., 1994). Signals that are provided by sensory neurons are essential to induce the expression of transcription factors specific to intrafusal muscle and are necessary for the differentiation of muscle spindle fibers.

\subsection{NRG signaling is essential for muscle spindle fiber differentiation}

The developing intrafusal muscle fibers of developing spindles specifically expresses several transcription factors, including the early growth response (Egr) family transcriptions factor, Egr3 (Tourtellotte and Milbrandt, 1998). Mice deficient in Egr3 
present with profound gait ataxia, due to the complete lack of spindle fibers; $E g r 3$ expression is essential for the differentiation of these structures (Tourtellotte and Milbrandt, 1998). The expression of Egr3 in certain cell types has been shown to be stimulated via NRG-1 mediated signal cascade (Sweeney et al., 2001). In vitro studies indicate that the NRG $\beta 1$ epidermal growth factor (EGF)-like domain is the minimal fragment of NRG sufficient to induce the immediate expression of genes and proteins required for spindle fiber differentiation, including Egr3 (Jacobson et al., 2004). In vivo, elimination of all isoforms of NRG results in severe impairment of muscle spindle development, as is evident by the lack of transcriptional markers, such as Egr3, as well as the absence of the characteristic annulospiral configuration of proprioceptive neurons surrounding the developing spindles (Hippenmeyer et al., 2002). More specifically, these findings also report that it is the immunoglobulin (Ig)-containing isoform of NRG, which is specifically expressed by proprioceptive neurons as opposed to other isoforms broadly expresses by most neurons, that is specifically required for muscle spindle formation (Hippenmeyer et al., 2002).

NRG signals through homo- and heterodimers of ErbB receptors. NRG is capable of binding directly to ErbB3 and ErbB4 (Fischbach and Rosen, 1997). Although ErbB3 demonstrates high affinity binding to NRG, it cannot autophosphorylate in response to NRG binding, which is necessary to transduce the signal, and it therefore forms functional heterodimers with either ErbB2 or ErbB4 by necessity (Burden and Yarden, 1997; Lemke, 1996). ErbB2 also forms heterodimers by necessity as it is incapable of directly binding to any of the NRG isoforms, it however, exhibits strong kinase activity (Lemke, 1996). The ErbB2-ErbB3 heterodimer is the most potent activator of the Ras- 
Erk pathway leading to proliferation and differentiation, and the phosphatidylinositol-3'kinase (PI3K)-Atk survival pathway (Altiok et al., 1997; Tansey et al., 1996). ErbB2 is essential in embryonic NRG-mediated spindle fiber differentiation and is also expressed by spindle fibers in adults (Andrechek et al., 2002; Leu et al., 2003). Ablation of ErbB2 in skeletal muscle results in gross proprioceptive deficits due to the complete lack of muscle spindle formation (Andrechek et al., 2002; Leu et al., 2003). ErbB3 and ErbB4 mediated signaling is also likely involved in the embryonic initiation of muscle spindles as both ErbB3 and ErbB4 expression has been reported in late embryonic development at sites where innervation occurs (Hippenmeyer et al., 2002).

\subsection{AGRN signaling results in aggregation of synapse-specific proteins}

In order for efficient NRG signaling through the ErbB receptors to occur between the in growing sensory neuron and the muscle, a stable synapse must be established, and the necessary postsynaptic proteins, the ErbB receptors included, must be recruited to the site of neural innervation. At neuromuscular junctions (NMJ)s, AGRN expressed by the in growing $\alpha$-motor neurons results in the clustering of acetylcholine receptors (AChRs) and other synaptic proteins such as LN- $\beta 2$, muscle specific kinase (MuSK), rapsyn, $\beta$ dystroglycan ( $\beta-\mathrm{DG})$ and utrophin (Meier et al., 1997). Clustering of AChRs at the NMJ will be used as a model to determine if the major contributing molecules also act in a similar manner in aggregating ErbB receptors at sensory synapses.

In skeletal muscle, the NMJs are highly specialized synapses that facilitate efficient signaling between motor neurons and proximal skeletal muscle. In embryonic 
development, before the motor neuron makes contact with the skeletal muscle, AChRs are dispersed diffusely across the muscle surface, but within hours of neural contact, the postsynaptic AChRs begin to form aggregates on the postsynaptic membrane (Bevan and Steinbach, 1977). Postsynaptic regions eventually pack densities of AChRs of $\sim 10$ $000 / \mathrm{um}^{2}$ compared to the unspecialized adjacent areas having an AChR density of 1000-fold less (Fertuck and Salpeter, 1974).

The clustering of AChRs and other synaptic proteins by AGRN commences when AGRN activates its functional receptor MuSK. AGRN initially binds low-density lipoprotein receptor four (Lrp4) (Kim et al., 2008; Zhang et al., 2008), which subsequently complexes with MuSK, resulting in MuSK phosphorylation (Glass et al., 1996; Hopf and Hoch, 1998). Docking protein 7 (Dok-7) and tumorous imaginal Discs 1 (Tid1) also complex with MuSK (1999; Linnoila et al., 2008; Okada et al., 2006), and are both required for efficient intracellular signal transduction; knockout mice for any of these genes is lethal at birth and there is an absence of AChR aggregates at postsynaptic terminals (DeChiara et al., 1996; Gautam et al., 1996; Linnoila et al., 2008; Okada et al., 2006). Dok-7 is involved in MuSK and AChR $\beta$-subunit phosphorylation and Tid1 demonstrates phosphorylation of AChR $\beta$-subunits and activation of small guanidine triphosphatases GTPases; both of these processes are necessary for interactions with rapsyn (Linnoila et al., 2008; Okada et al., 2006). Rapsyn is associated with AChRs, connects AChRs to the cytoskeleton and is required in AChR clustering (Fuhrer et al., 1999). A MuSK-rapsyn interaction is mediated though a rapsyn-associated transmembrane linker (RATL) (Apel et al., 1997). Ultimately, the activation of MuSK results in the phosphorylation of the $\beta$-subunit of the AChR, which is a necessary 
requirement for enhancing the linkage of the AChRs to the cytoskeleton (Borges and Ferns, 2001; Wallace et al., 1991).

Numerous other synapse-specific proteins are also aggregated by AGRN and colocalize with AChRs, included in these are the NRG receptors ErbB2 and ErbB3 (Meier et al., 1997; Rimer et al., 1998). ErbB4 has been shown to be associated with the adaptor protein syntrophin, which is also localized to NMJs (Garcia et al., 2000). Based in this, perhaps AGRN, which has been shown to aggregate ErbB2, ErbB3 and ErbB4 to AChR cluster regions (Meier et al., 1997; Rimer et al., 1998), is also involved in the accumulation of ErbB receptors to the synapses formed between propriocetive sensory neurons and muscle.

In the developing nervous system, AGRN is expressed in specific patterns at specific developmental time points. Both primordial motor and sensory neuron derivatives in the rat and chick express high levels of AGRN transcripts (Ma et al., 1994; Ma et al., 1995; Stone and Nikolics, 1995). Although AGRN is the product of a single gene, many different isoforms exist (Rupp et al., 1991). These are generated through alternative transcriptional start sites (Burgess et al., 2000; Denzer et al., 1995), as well as extensive combinations of alternative splice sites contained in the C-terminal end (Burgess et al., 1999; Ferns et al., 1993; Hoch et al., 1993). The primary AGRN transcript contains three potential alternative splice sites, denoted $\mathrm{X}, \mathrm{Y}$, and $\mathrm{Z}$ in rodents and $\mathrm{A}, \mathrm{B}$ in chicks (Hoch et al., 1993; Ruegg et al., 1992). A 12-amino acid insert at the X site appears to have no effect on the ability of AGRN to cluster AChRs (Ferns et al., 1992). It is inserts of 8,11 , or 19-amino acids at the Z-spice site that demonstrate the greatest effect in the ability of AGRN to assemble aggregates of AChRs (Ferns et al., 1992). The 
highly active Z-site insert containing isoforms are expressed exclusively by motor neurons in the developing nervous system (Hoch et al., 1993; Stone and Nikolics, 1995), and are highly expressed during the developmental time period that corresponds to synaptogenesis (Stone and Nikolics, 1995). Although all isoforms of AGRN exert some AChR clustering ability, it is the Z-splice variants (or B-splice variants in chick) that exhibit the most potent aggregating capacity (Ferns et al., 1992; Ferns et al., 1993). Interestingly, the presence of a 4-amino acid insert at the Y-splice site does increase the AChR clustering activity by 2-fold, when there is no Z-insert present (Ferns et al., 1993). This isoform, $\operatorname{AGRN}_{(4,0)}$, is extensively expressed by rat sensory neurons in the dorsal root ganglion (DRG) at E(15) (Stone and Nikolics, 1995), just prior to the initial appearance of nascent muscle spindle fibers on E(17) (Zelena, 1994).

Additionally, at the NMJ ErbB2 and ErbB3 are also co-localized to these AGRNinduced postsynaptic clusters, presumably through association with syntrophin (Garcia et al., 2000; Meier et al., 1997; Rimer et al., 1998). This aggregation of ErbB receptors is also observed in myotube tissue cultures that constitutively express MuSK (Jones et al., 1999). Furthermore, knockout mice that are MuSK or AGRN deficient fail to assemble aggregates of ErbB receptors (DeChiara et al., 1996; Gautam et al., 1996). In order to ensure efficient transduction of NRG signaling leading to activation of Egr3 expression, which subsequently induce a network of spindle fiber specific genes (Albert et al., 2005), ErbB receptors must be in close proximity to the inductive signal. AGRN is expressed in both motor and sensory neurons as evident by expression in embryonic spinal cord primordial motor neurons as well as DRG in chicken embryos (Ma et al., 1995). Rat DRG express exceedingly high levels of $\mathrm{AGRN}_{(4,0)}$ at E13 through E15 (Stone and 
Nikolics, 1995). Perhaps AGRN expressed by in growing sensory Ia afferents during development is a source of AGRN that is adequate to cause the aggregation of the ErbB receptors and allow for effective NRG signaling resulting in induction of Egr3 and ultimately spindle fiber differentiation.

\section{$1.5 \alpha-D G$ the transmembrane link}

AGRN acts to aggregate AChRs and other synaptic proteins to the NMJ in a fast and effective manner through activation of MuSK (Glass et al., 1996; Hopf and Hoch, 1998). However, AGRN also binds directly to $\alpha$-DG (Bowe et al., 1994; Campanelli et al., 1994; Gee et al., 1994; Sugiyama et al., 1994). $\alpha$-DG is a highly glycosylated, peripheral membrane protein and is part of the dystrophin-associated glycoprotein complex (DGC); disruption or absence of this complex of proteins can result in any number of muscular dystrophies (Ervasti et al., 1990; Ibraghimov-Beskrovnaya et al., 1992). $\alpha-D G$ is the product of a single gene (dag1) that is initially expressed as a propeptide and subsequently post-translationally cleaved generating two distinct subunits, $\alpha$ DG and the transmembrane $\beta$-DG (Holt et al., 2000; Ibraghimov-Beskrovnaya et al., 1992). $\alpha$ - and $\beta$-DG are associated in a non-covalent manner and this interaction creates the core of the DGC effectively linking the extracellular matrix (ECM) to the underlying cytoskeleton, a link that is essential to maintaining the integrity of skeletal muscle and protecting it from the mechanical stress caused by contraction (Ervasti and Campbell, 1993; Henry and Campbell, 1996; Michele and Campbell, 2003). At NMJs in skeletal muscle, $\beta$-DG associates with rapsyn and colocalizes with AChRs at newly forming synaptic clusters (Bartoli et al., 2001; Cartaud et al., 1998; Cohen et al., 1995). 
$\alpha$-DG was once thought to be the functional AGRN receptor (Gee et al., 1994). AGRN does indeed bind to $\alpha$-DG via its Laminin G-like (LG) modules (Hohenester et al., 1999), however, $\alpha$-DG does not demonstrate the kinase activity required for phosphorylation of the AChR $\beta$-subunit, which is necessary for enhanced anchoring of the AChRs to the cytoskeleton (Borges and Ferns, 2001; Wallace et al., 1991). $\alpha$-DG binds to the active neural AGRN isoform, but also binds with high affinity to the other AGRN isoforms that do not have a significant affect on AChR clustering (Gesemann et al., 1996; Hopf and Hoch, 1996; Sugiyama et al., 1994). Other evidence that suggests a role for $\alpha$-DG in consolidation and maintenance as opposed to the initial signal in NMJ formation is that $\alpha$-DG deficient myotubes do form NMJs, and although they are altered in morphology, clusters of AChRs are still present (Cote et al., 1999; Grady et al., 2000; Jacobson et al., 1998). $\alpha$-DG functions in AGRN-induced AChR clustering independent of MuSK, as AGRN-MuSK signaling is not substantially inhibited in muscle cell lines deficient in $\alpha$-DG (Jacobson et al., 1998). In competitive binding experiments disrupting the ARGN- $\alpha-\mathrm{DG}$ interaction, such as treatment with muscle AGRN, which binds $\alpha$-DG with a 10-fold higher affinity than neural AGRN (Campanelli et al., 1994; Gesemann et al., 1996; Sugiyama et al., 1994), or with anti- $\alpha$-DG antibodies, results in no significant decrease in phosphorylation of MuSK or the downstream phosphorylation of the AChR $\beta$-subunit (Jacobson et al., 1998). $\alpha$-DG is now thought to work downstream of MuSK activation in AGRN-induced AChR clustering where it works to regulate the stability of AChR aggregates. First AGRN-induced MuSK activation mediates the formation of AChR microclusters (Froehner et al., 1990; Phillips et al., 1991), which are then subsequently condensed and then maintained as larger clusters (Figure 1; (Cohen et al., 
1995; Jacobson et al., 1998). Unstable and diffuse AChR clusters are observed in muscle fibers that are deficient in $\alpha$-DG (Cote et al., 1999; Grady et al., 2000; Jacobson et al., 2001), as well as a significant reduction in LN, perlecan and acetylcholine esterase (AChE) at these $\alpha$-DG deficient NMJs (Cote et al., 1999; Jacobson et al., 2001). These findings suggest a role for $\alpha-D G$ in the stabilization and condensation of AChR clusters.

Since $\alpha-D G$ is involved in stabilizing and condensing aggregates of AChRs (Cote et al., 1999; Jacobson et al., 2001), which have also been shown to colocalize with ErbB receptors at NMJs (Jones et al., 1999; Meier et al., 1997), perhaps $\alpha$-DG is of importance in clustering ErbB receptors at sensory synapse. Another extracellular matrix protein that binds $\alpha$-DG is LN, perhaps it too works to facilitate NRG-ErbB signaling by involvement in clustering ErbB receptors. 


\section{Figure 1: Clustering of AChRs at the NMJ}

AGRN secreted from motor neurons binds to Lrp4, which is complexed with MuSK and induces MuSK phosphotyrosylation. Dok-7 is subsequently phosphorylated and complexes with Tid1, which in turn works to activate small GTPases (not shown), ultimately phosphorylating the AChR $\beta$-subunits. This cascade results in small microclusters of AChRs at nascent NMJs. The basal lamina protein LN then acts to condense the microclusters into large, mature, stable clusters through binding to $\alpha$-DG and LN self-polymerization via the short arms pt LN cruciform (A). AGRN can also cluster AChRs by binding to $\alpha$-DG. This may occur as a result of up steam MuSK activation and clustering $\alpha$-DG into clusters (B). LN also demonstrates the ability to cluster AChRs. High concentrations of LN added to myotubes in vitro cause AChRs to aggregate. This has been shown to occur independent of MuSK activation by AGRN. LN clusters by binding $\alpha$-DG and then binding to other $\mathrm{LN}$ via homophilic interactions between short arms (B).

Rap $=$ rapsyn, $P=$ phophotyrosine phosphorylation 


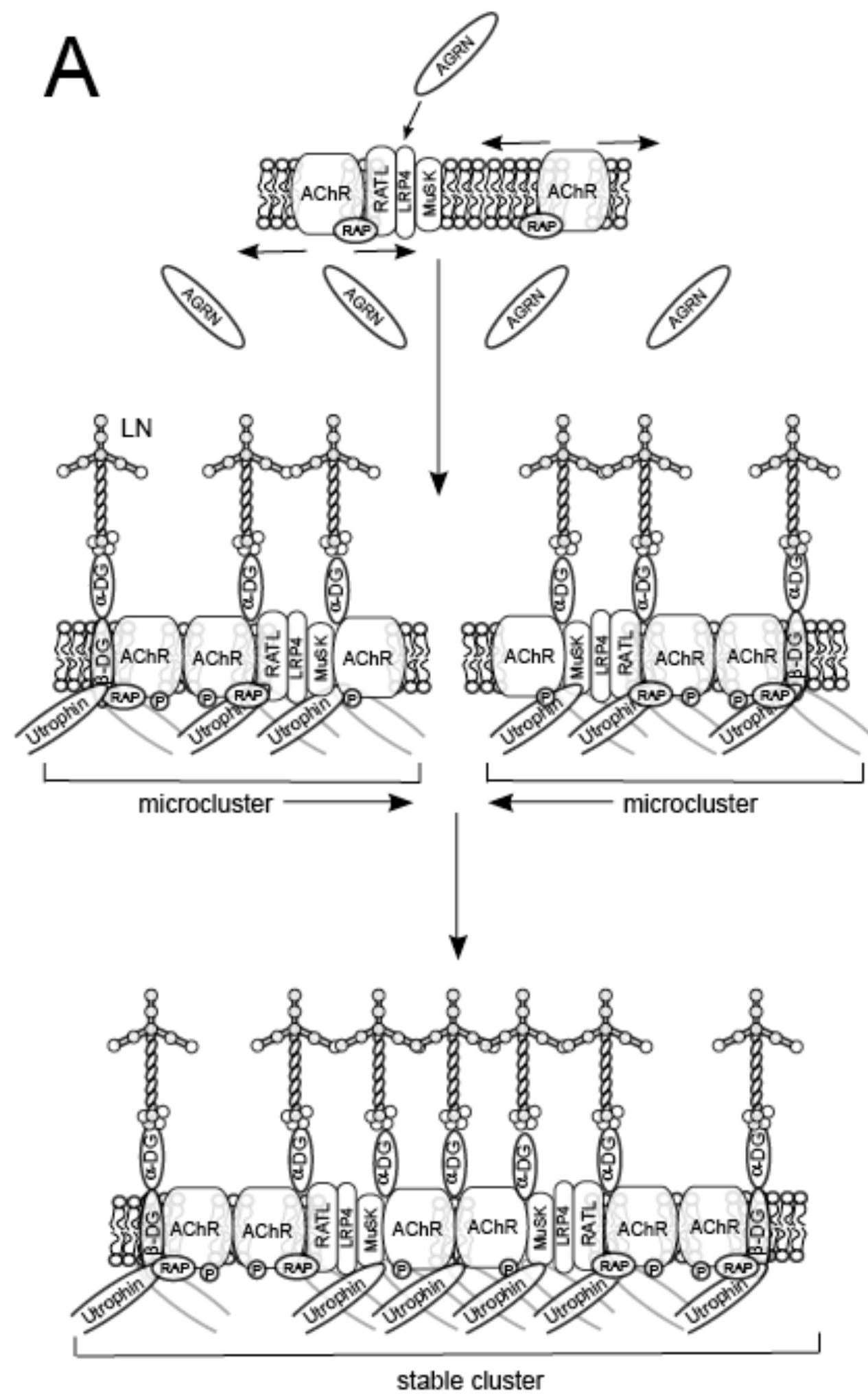


B
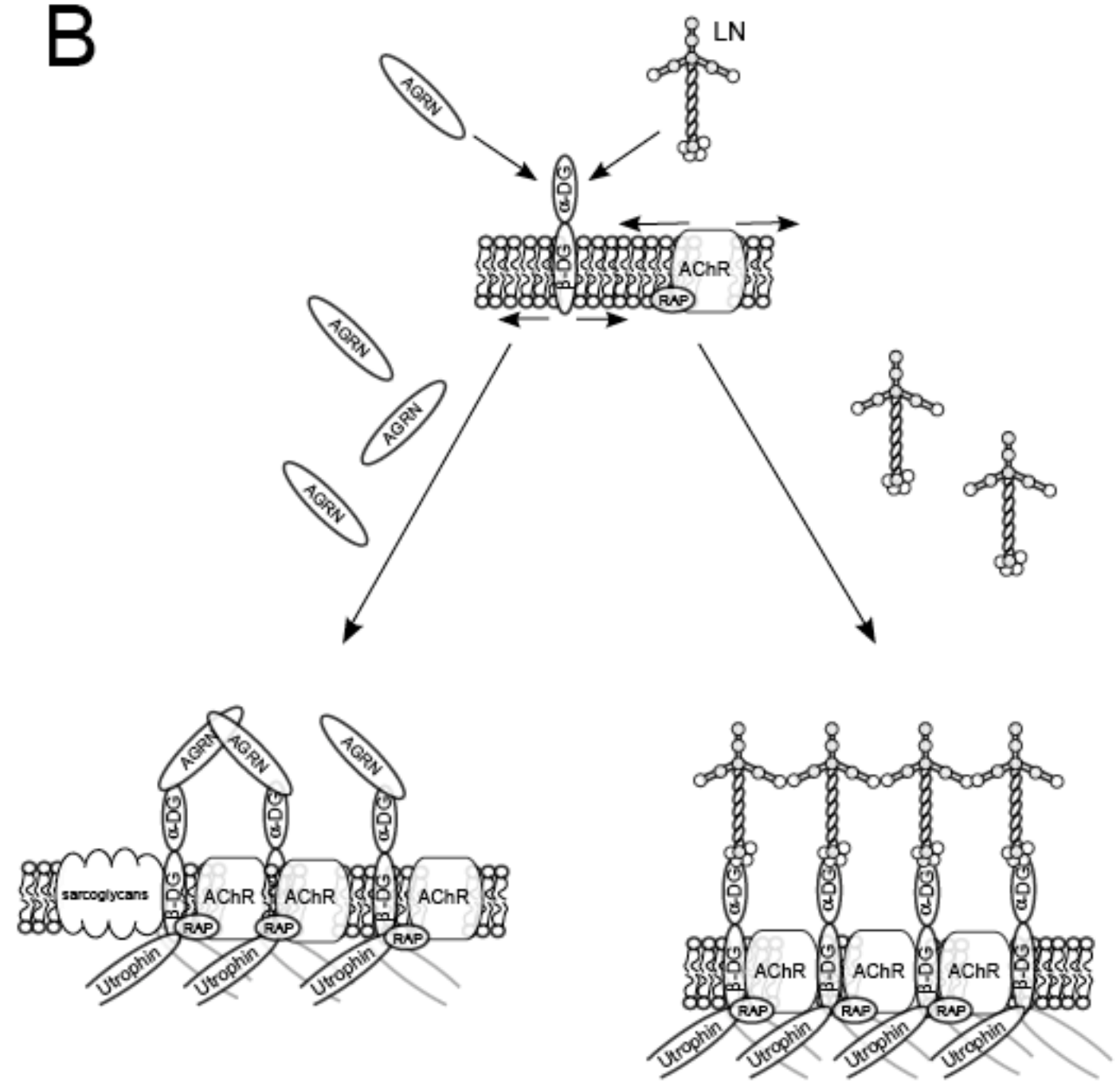


\section{6 $L N$ is a condenser and stabilizer of AChR synaptic clusters}

The LNs are part of a large family of heterotrimeric proteins comprised of at least 12 isoforms (Aumailley and Smyth, 1998; Koch et al., 1999). LNs can polymerize into large sheet-like structures and along with collagen IV, perlecan and nidogen/entactin constitute a large proportion of the ECM (Timpl and Brown, 1996). Specific LN isoforms, $\mathrm{LN}-1(\alpha 1 \beta 1 \gamma 1)$ and $\mathrm{LN}-2(\alpha 2 \beta 1 \gamma 1)$ demonstrate high affinity binding to $\alpha$-DG, while it is the $\mathrm{LN}-2(\alpha 2 \beta 1 \gamma 1)$ isoform that provides the critical like of the cytoskeleton to the ECM in skeletal muscle, as mutations to the $\mathrm{LN}-2 \alpha 2$ (also referred to as merosin) chain results in congenital muscular dystrophies (Campbell, 1995). LN (now referring to LN-2 $\alpha 2$ chain containing isoforms from this point on) are crucifix-shaped molecules with the long arm $\alpha$ chain containing a tandem of five LG modules at the C-terminal end which contain the $\alpha$-DG binding sites (Figure 1; (Ervasti and Campbell, 1993; Gee et al., 1993; Smalheiser, 1993). This binding is calcium dependent, just as $\alpha$-DG-AGRN binding is also through LG modules and is calcium dependent (Bowe et al., 1994; Campanelli et al., 1994; Gee et al., 1994; Sugiyama et al., 1994). The LG modules of LN likely bind to the central, highly glycosylated, mucin-like region, which is rich in negatively charged oligosaccharides (Durbeej et al., 1998).

During synaptic differentiation in skeletal muscle, as previously explained, AGRN from in growing motor neurons activates MuSK, which in turn phosphorylates the AChR $\beta$-chains resulting in aggregates of AChRs forming microclusters (Figure 1;

(Froehner et al., 1990; Phillips et al., 1991). LN binding to $\alpha$-DG then acts to stabilize and condense these microaggregates into larger clusters (Figure 1; (Cohen et al., 1995; 
Jacobson et al., 1998). MuSK activation leading to AChR clustering results in the coclustering of $\alpha$-DG to these developing synapses (Cohen et al., 1995), in a rapsyndependent manner (Apel et al., 1995). This recruitment of $\alpha-D G$ to these clusters acts as a scaffold that works to immobilize AChRs and other synaptic proteins to these developing synapses via $\alpha$-DG-LN interactions (Apel et al., 1997; Carbonetto and Lindenbaum, 1995; Ibraghimov-Beskrovnaya et al., 1992). LN itself has been shown to self-assemble in solution by homophilic interactions between its short arms (Yurchenco et al., 1992); this phenomenon has also been observed on the surface of muscle cells (Cohen et al., 1997). Interestingly, several studies have demonstrated that LN addition to myotube cultures can actually promote the accumulation of AChRs into large clusters, via binding to $\alpha$-DG, independent of AGRN (Montanaro et al., 1998; Sugiyama et al., 1997). Since $L N-\alpha-D G$ interactions are an important part of $A C h R$ clustering in both a MuSK-AGRN dependent and independent manner, conceivably these interactions may play an important role in the aggregation of ErbB receptors at developing sensory synapses and facilitate efficient NRG signaling.

\subsection{Research objectives}

Experiments were designed to establish whether the key proteins involved in the clustering of AChRs in developing motor synapses, AGRN, LN and $\alpha$-DG, are also important in sensory synapses formation. Given that AGRN and LN are capable of clustering AChRs at motor synapses (McMahan, 1990; Montanaro et al., 1998; Sugiyama et al., 1997), and that AGRN also aggregates ErbB receptors to these clusters (DeChiara et al., 1996; Gautam et al., 1996), initially it was determined whether addition of AGRN and LN addition to myotube cultures had any affect on the expression of the transcription 
factor Egr3, which has previously been used as a marker for muscle spindle differentiation (Hippenmeyer et al., 2002). Egr3 levels were monitored using immunoblot analysis and both molecules proved effective in inducing Erg3 expression. To investigate $\alpha$-DG's role in this process complimentary methods were used. In the first instance, an $\alpha$ DG silenced muscle cell line was used and in the second instance, basal lamina/ $\alpha-D G$ interactions were blocked, using an anti- $\alpha$-DG antibody. Again, Egr3 levels were evaluated by immunoblot analysis. In both cases treatments resulted in a dramatic increase in Egr3 expression, which was substantially decreased in $\alpha$-DG deficient myotubes and myotubes blocked with anti- $\alpha-D G$ antibodies. Blocking experiments with NRG and ErbB inhibitors were used to determine if LN induced Egr3 expression was occurring as a result of NRG-ErbB signaling and not in some other novel pathway. Inhibiting NRG and ErbB signaling did indeed reduce the expression of Egr3 induced by LN, indicating LN induction of Egr3 does occur via NRG-ErbB signaling. Furthermore, immunocytochemistry was used to determine the levels of muscle spindle fiber specific slow developmental myosin heavy chain (sd-MyHC) expression. However, no change in sd-MyHC was observed. Finally, the effect of LN on both AChR and ErbB3 clustering was examined using direct and indirect immunocytochemistry. Taken together, analysis of the results from these experiments revealed that indeed AGRN, LN and $\alpha$-DG influence Egr3 levels and therefore may play an important role in spindle fiber differentiation. Based on these results, a role for AGRN and LN binding to $\alpha$-DG at sensory synapses that acts upstream of NRG-ErbB signaling to allow the effective transmission of NRG-ErbB signaling to occur and stimulate Egr3 induction is inferred. This likely occurs as a consequence of AGRN and LN's ability to stimulate clustering of 
the ErbB receptors by inducing physical changes in the basal lamina resulting in the immobilization of ErbB receptors and other synaptic proteins to these regions. 


\section{Chapter 2}

\section{Materials and Methods}

\subsection{Cell culture}

Human primary myoblasts were provided as a gift from H. Blau (Stanford

University, Stanford, CA). Cells were cultured on plastic tissue culture plates coated with $0.1 \%$ collagen in HAMS F-10 medium (Wisent, St-Bruno, QC) supplemented with 15\% fetal bovine serum (FBS; Wisent, St-Bruno, QC), $100 \mathrm{U} / \mathrm{ml}$ penicillin/streptomycin (Wisent, St-Bruno, QC), and 0.5\% chick embryo extract (Sera Laboratories International, UK). Cultures were grown at $37^{\circ} \mathrm{C}$ in $5 \% \mathrm{CO}_{2}$ and $95 \%$ humidity. Myoblasts were fused to myotubes after reaching confluence by maintaining cultures in Dulbecco's modified Eagle's medium (DMEM; Wisent, St-Bruno, QC) high glucose formula supplemented with 2\% horse serum (Wisent, St-Bruno, QC), 1\% insulin-transferrin-selenium (Mediatech Inc., Manassas, VA), and $100 \mathrm{U} / \mathrm{ml}$ penicillin/streptomycin (Wisent, StBruno, QC). Fusion of myoblasts to myotubes typically took 3-4 days.

The C2C12 myoblast cell line was obtained from the American Type Culture Collection (ATCC; CRL-1772). Myoblasts were seeded on plastic tissue culture plates and grown in DMEM low glucose medium (Wisent, St-Bruno, QC) supplemented with $20 \% \mathrm{FBS}$ and $100 \mathrm{U} / \mathrm{ml}$ penicillin/streptomycin at $37^{\circ} \mathrm{C}, 95 \%$ humidity in $8 \% \mathrm{CO}_{2}$. Fusion of myoblasts was induced by changing to fusion medium consisting of DMEM high glucose (Wisent, St-Bruno, QC) supplemented with 2\% horse serum (Wisent, St- 
Bruno, QC) and $100 \mathrm{U} / \mathrm{ml}$ penicillin/streptomycin. Fusion of myoblasts to myotubes typically took 3-4 days.

The $\alpha$-DG-silenced cell line, 11E myoblasts, were a generous gift from S. Carbonetto (McGill University, Montreal, QC). The 11E cultures were maintained as previously described (Jacobson et al., 1998). Briefly, 11E cells were derived from C2C12 myoblasts and grown similarly, with the addition of the antibiotic G418 sulfate (70 $\mu \mathrm{g} / \mathrm{ml}$; Wisent, St-Bruno, QC) to the growth medium. Fusion to myotubes usually required 5-6 days in fusion medium.

Mouse-anti-slow developmental myosin heavy chain (sd-MyHC) specific monoclonal antibody producing hybridomas were obtained from The Developmental Studies Hybridoma Bank (University of Iowa, Iowa City, IA). Hybridomas were grown in Iscove's DMEM (Wisent, St-Bruno, QC) supplemented with 20\% heat inactivated FBS and Gentamycin sulfate (50 $\mu \mathrm{g} / \mathrm{ml}$; Wisent, St-Bruno, QC). Culture growth conditions were $37^{\circ} \mathrm{C}, 5 \% \mathrm{CO}_{2}$ and $95 \%$ humidity. To collect antibodies, culture medium was gently centrifuged $(52 \times \mathrm{g})$ to remove cells. The supernatant was then collected and stored at $4^{\circ} \mathrm{C}$. To determine the approximate concentration of antibodies, dilutions of the supernatant were electrophoresed on a $10 \%$ sodium dodecyl sulfate polyacylamide gel (SDS-PAGE) and transferred to nitrocellulose and immunoblotted (see section 2.5). Antibodies were visualized using an anti-mouse antisera conjugated to horseradish peroxidase (1:2000; GE Health Care, UK). Immunoblot results are shown in the Appendix (see Figure A1). 


\subsection{Treatments and blocking experiments}

\subsubsection{Culture treatments for immunoblot analysis}

Time course experiments were preformed by refreshing the fusion medium of fused C2C12 myotubes and adding NRG (1 nM; Shenandoah Biotechnology Inc., Warwick, PA), NRG with AGRN (500 pM; rrC-Ag 3,4,8, R \& D Systems, Minneapolis, $\mathrm{MN}$ ), and AGRN alone to cultures. Treatments were added to culture plates at 1, 3, 6, 12 and 24 hours prior to protein extraction. A non-treated control was also prepared at the 24-hour time point. The concentrations of the treatments are consistent in all experiments unless stated differently.

In experiments were $\mathrm{LN}$ was used, it was added to $\mathrm{C} 2 \mathrm{C} 12$ and $11 \mathrm{E}$ cultures at a final concentration of $50 \mathrm{nM}$ (Millipore Corp., Billerica, MA). The LN treatment along with NRG was added to cultures four hours prior to protein extraction. An untreated control was also prepared at this time.

\subsubsection{Blocking experiments}

$\alpha$-DG was blocked with the monoclonal antibody 11H6 (sc-53987; Santa Cruz Biotechnology, Santa Cruz, CA). This antibody was added to C2C12 myotubes one hour prior to treatments with $\mathrm{NRG}, \mathrm{LN}$ and an untreated control at a dilution of 1:50 in fusion medium.

NRG function was blocked using an anti-NRG1- $\beta 1$ EGF domain-neutralizing antibody (AF-396-NA; R \& D Systems, Minneapolis, MN). It was added at the time of treatment with NRG, LN and an untreated control at a concentration of $2 \mu \mathrm{g} / \mathrm{ml}$. Treatments were added two hours before protein extraction. 
Blocking ErbB receptor signaling was achieved by using Tyrphostin AG825. Tyrphostin AG825 (50 mM; Cayman Chemical, Ann Arbor, MI) was added to cultures and incubated overnight before the addition of treatments with NRG, LN and an untreated control. These treatments were added four hours before protein extraction.

\subsubsection{Culture treatments for immunofluorescence}

All treatments of $\mathrm{C} 2 \mathrm{C} 12$ and $11 \mathrm{E}$ cultures prepared for immunofluorescence were incubated overnight (18 hrs) prior to preparation of cultures for fixation. All concentrations of treatments were the same as previously stated for western blotting purposes.

\subsection{Protein extractions}

Following treatment of fused myotubes, cultures were washed twice with Dublecco's Phosphate-buffered Saline (D-PBS; Wisent, St-Bruno, QC) solution and collected in D-PBS and centrifuged at $4{ }^{\circ} \mathrm{C}$ and $470 \times$ g. The pellet of cells was resuspended in protein extraction buffer $[25 \mathrm{mM}$ Tris- $\mathrm{HCl} \mathrm{pH}$ 7.5, $25 \mathrm{mM}$ Glycine, 150 $\mathrm{mM} \mathrm{NaCl}, 5 \mathrm{mM}$ EDTA, 1\% Triton X-100, and 1x Complete Protease Inhibitor Cocktail (Roche, Mississauga ON)] and incubated on ice for 15 minutes and then centrifuged at $16000 \times \mathrm{g}$ at $4^{\circ} \mathrm{C}$. The supernatant was collected and used for immunoblot analysis.

\subsection{Immunoblot analysis}

Protein extracts were quantified using the Bio-Rad Protein Assay (Bio-Rad Laboratories, Mississauga, ON) to ensure equal protein sample loading. Immunoblots were performed according to methods previously described (Jacobson et al., 1998). In 
brief, quantified protein extracts were electrophoresed using $8 \%$ SDS-PAGE and subsequently transferred to a nitrocellulose membrane. Ponseau $\mathrm{S}$ was used to ensure successful transfer of proteins as well as verify equal loading. Following electrophoretic transfer to nitrocellulose membranes were blocked for one hour at room temperature in blocking buffer (5\% skim milk powder in $10 \mathrm{mM}$ Tris- $\mathrm{HCl} \mathrm{pH} \mathrm{7.5,} 0.15 \mathrm{M} \mathrm{NaCl}$ and $0.1 \%$ Tween 20). Membranes were then incubated with primary antibody [Egr3 (1:400), Santa Cruz Biotechnology, Santa Cruz, CA; $\alpha$-DG (1:1000), sc-53987 Santa Cruz Biotechnology, Santa Cruz, CA)] in blocking buffer for one hour at room temperature. Horseradish peroxidase (HRP)-conjugated secondary antibodies were used to visualize protein-specific binding and were diluted in blocking buffer and incubated for one hour at room temperature [(1:2000), GE Health Care, UK]. Chemiluminescence was detected using the combination of ECL western blotting substrate (Peirce, Rockford, IL) and autoradiography film (Labscientific Inc., Livingston, NJ).

An extra protein sample was also electrophoresed on each gel for use as a secondary antibody control lane. This lane contained quantified protein extract from NRG treated $\mathrm{C} 2 \mathrm{C} 12$ cells and was left in blocking solution while all other lanes were incubated in primary antibody. Incubation in conjugated secondary antibody solution and detection was carried out along with all test lanes.

To quantify protein expression levels, densitometric analysis was carried out on sub-saturated film exposures using the gel analysis macro included in the NIH image software package available at: ftp://rsbweb.nih.gov/pub/nih-image. All values were expressed as a percentage of the control value (zero time point) in order to average several independent experiments. 


\subsection{Immunofluorescence}

Myotubes used for immunofluorescence were grown on acid washed (bathed in $66 \%$ concentrated nitric acid and 33\% concentrated hydrochloric acid for 2 hours and rinsed in deionized water for 30 minutes) glass cover slips, which were subsequently coated with $0.1 \%$ gelatin. For experiments were $\alpha$-bungarotoxin ( $\alpha$-BTX) was used to observe AChR clusters, rhodamine-labeled $\alpha$-BTX ( $0.2 \mu \mathrm{g} / \mathrm{ml}$; Molecular Probes) was added to treated cultures one hour prior to fixation. To verify that the rhodamine-labeled $\alpha$-BTX indeed binds specifically to AChRs, a control was prepared by adding a 10 -fold excess of unlabeled $\alpha$-BTX ( $2 \mu \mathrm{g} / \mathrm{ml}$; Molecular Probes) to a culture plate 30 minutes before the addition of rhodamine-labeled $\alpha$-BTX. Following all treatments, cultures were washed twice with PBS and then fixed either with $2 \%$ paraformaldehyde or $70 \%$ ice-cold ethanol.

Experiments where ErbB3 and AChR clusters were examined; cultures were fixed in $2 \%$ paraformaldehyde for 20 minutes and permeablized in $0.3 \%$ Triton $\mathrm{x}-100$ in PBS for 10 minutes. When looking at the levels of sd-MyHC, cultures were fixed in $70 \%$ ice-cold ethanol at $-20^{\circ} \mathrm{C}$ for 15 minutes and permeablized in $0.1 \%$ Triton $\mathrm{x}-100$ in PBS for 10 minutes. After permeabilzation, cultures were incubated for one hour in blocking buffer (10\% horse serum in PBS). Primary antibodies were diluted in blocking buffer and incubated for one hour at room temperature [anti-ErbB3 (1:200); Santa Cruz Biotechnology, Santa Cruz, CA] or at $4^{\circ} \mathrm{C}$ overnight [anti-sd-MyHC (1:2), The Developmental Studies Hybridoma Bank, IA]. Cultures were washed five times with PBS; specific binding was detected with fluorescein-conjugated secondary antiserums [anti-rabbit and anti-mouse, both used at 1:2000, (Molecular Probes)]. Cultures were 
washed five times with PBS before the addition of ProLong Gold antifade reagent (Molecular Probes) and the cover slips mounted onto slides. Fluorescence was observed using a Zeiss Axiovert 200 inverted microscope (Zeiss, Burnaby, BC) at a final magnification of $400 \times$ for examination of ErbB3 and $\mathrm{AChR}$ clusters and inspection of myotubes for sd-MyHC. To record actual observed differences in fluorescence, experimental and control samples were photographed under identical conditions using a Qimaging retiga 1494 digital camera in combination with OpenLab software (Improvision, Waltham, MA).

\subsection{Quantification of AChR and ErbB3 colocalization}

$\mathrm{C} 2 \mathrm{C} 12$ and $11 \mathrm{E}$ myotubes were treated overnight (18 hours) with NRG and LN as well as an untreated control. AChR and ErbB3 clusters were visualized on paraformaldehyde-fixed myotubes with rhodamine-labeled $\alpha$-BTX and indirect fluorescence using ErbB3-specific antibodies. Myotubes were magnified $400 \times$ using the Zeiss Axiovert 200 inverted microscope (Zeiss, Burnaby, BC) and 10 random, nonoverlapping fields of view were photographed for quantification. Quantification of overlapping $\mathrm{AChR}$ and ErbB3 aggregates was determined by visual inspection of photographs imported into Adobe Photoshop (Adobe Photoshop CS Version 8.0). In Photoshop, the contrast of the photographs was adjusted for optimal viewing, and any and all photographic manipulations made in Photoshop were kept consistent between all treatments and experiments. Visible clusters of rhodamine-labeled $\alpha$-BTX bound to AChRs were counted and compared to the immunofluorescence pattern produced by staining for ErbB3. When $\geq 50 \%$ of the fluorescence of similar size and shape corresponded to that of the $\alpha$-BTX fluorescence pattern, an overlap was considered. To 
account for differences in cell densities, the average number of AChR aggregates per myotube was also recorded. This was accomplished by counting the number of AChR clusters and the total number of myotubes in that field of view. The ratio of AChR clusters to the number of myotubes was then calculated. 


\section{Chapter 3}

\section{Results}

\subsection{Induction of Egr3 by AGRN and LN}

Previous studies have used the induction of the transcription factor Erg3 as a molecular marker to identify the early initiation stages of muscle spindle fiber differentiation (Hippenmeyer et al., 2002). It has also been established that NRG provided by the in growing Ia sensory neuron induces the production of Egr3, which is necessary for muscle spindle development (Hippenmeyer et al., 2002; Jacobson et al., 2004; Tourtellotte and Milbrandt, 1998). NRG signals through the ErbB family of tyrosine kinase receptors and in order for an efficient transmission of signal into the growing muscle to occur the ErbB receptors must be concentrated and in close proximity to the site of contact. AGRN and LN are involved in clustering and consolidation of AChR clusters at the NMJ (McMahan, 1990; Montanaro et al., 1998; Sugiyama et al., 1997). It has been shown that ErbB2 and ErbB3 colocalize with AGRN-induced AChR clusters (Meier et al., 1997; Rimer et al., 1998). Considering these findings, this study investigated whether AGRN and LN influence the induction of Egr3.

To determine whether AGRN and LN are involved, the mouse C2C12 myotube cell line was utilized. This line has been characterized extensively in studies of postsynaptic differentiation and is relatively easy to grow, readily available from the ATCC, and is similar in many respects to human myotubes. This was important, as more direct connections and possible future medical implications to human health may be inferred as a consequence of data attained from this study. For this reason, initial 


\section{Figure 2: Induction of Egr3 in C2C12 mouse myotubes}

Shown here is an immunoblot for Egr3 following treatments with NRG, NRG and AGRN, and AGRN alone in Human and mouse myotubes. The levels of Egr3 protein were increased in $\mathrm{C} 2 \mathrm{C} 12$ mouse myotubes when treated for 2 hours with $\mathrm{NRG}, \mathrm{NRG}$ and AGRN and AGRN as compared to the basal level of Egr3 in the untreated control. This particular anti-Egr3 antibody (Santa Cruz Biotechnology, Santa Cruz, CA) recognized Egr3 in C2C12 mouse myotube whole protein extracts as opposed to human myotube protein extracts that were prepared the same way and cultures given the same treatments for the same duration as well as a shorter incubation. 


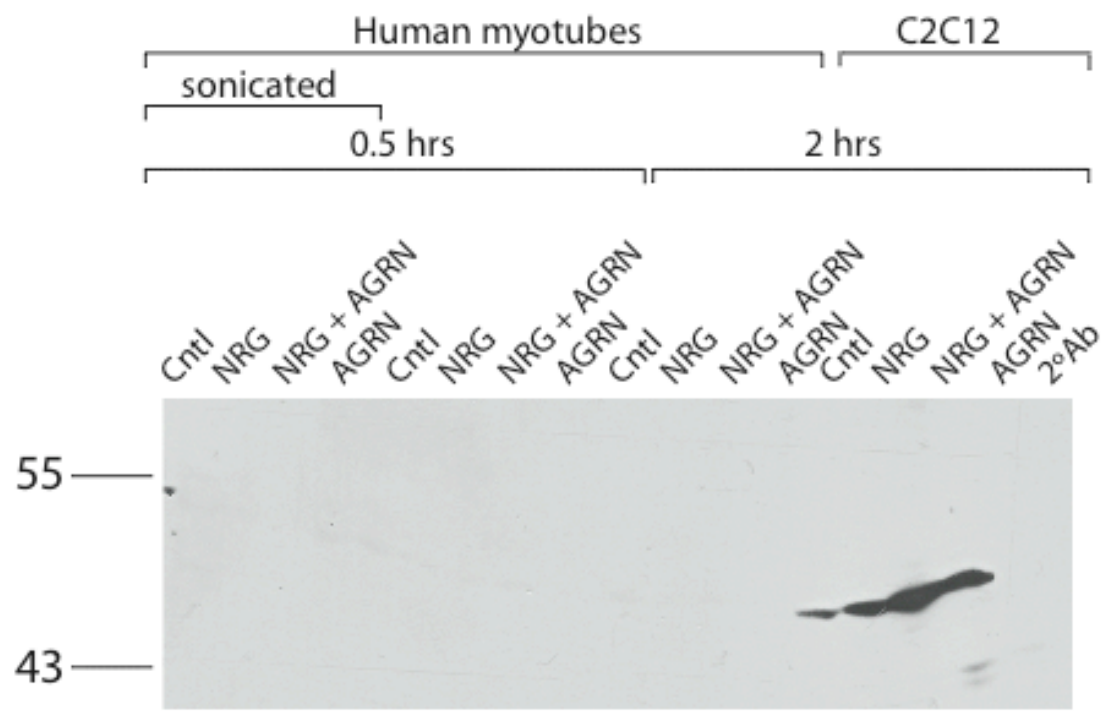


experiments were carried out in a human primary myotube cell line. Unfortunately, the commercially available anti-Egr3 polyclonal antibody readily recognizes the Egr3 from mouse myotube protein extracts far better than that of the human myotube protein extracts when used for western blotting (Figure 2). The direct comparison of human myotubes and $\mathrm{C} 2 \mathrm{C} 12$ mouse myotubes both treated for 2 hours with a control, NRG, NRG + AGRN and AGRN alone exhibited a very different result after western blotting. There is no visible band at $46 \mathrm{kDa}$ in the lanes where human myotube extracts were blotted, in contrast to the lanes corresponding to $\mathrm{C} 2 \mathrm{C} 12$ extracts where Egr3 protein was clearly recognized by the antibody (Figure 2). Even a shorter incubation time of human myotubes with the same treatments resulted in the absence of banding at $46 \mathrm{kDa}$ where Egr3 should be recognized by immunobloting. Due to the absence of obvious Egr3 immunoreactivity in the human primary cells further studies were restricted to $\mathrm{C} 2 \mathrm{C} 12$ myotubes.

To determine the optimal time point to look at Erg3 induction in further experiments, a 24-hour time course was completed. C2C12 myotubes were treated for 1, 3, 6, 12 and 24-hours with NRG, NRG + AGRN and AGRN and protein lysates were prepared for immunoblotting. Egr3 protein levels were determined by immunoblot, quantified by densitometry, and the resulting values normalized to the untreated control. All values were expressed as a percentage of the control so that values could be averaged across several independent experiments. Immunoblot analysis revealed that NRG induced Egr3 levels reached the highest point by 3 hours and remained high over the 24-hour period (Figure 3), which is consistent with values obtained by Jacobson et al., 2001. 


\section{Figure 3: NRG and AGRN induction of Egr3 over 24 hours}

Representative Western blot for Egr3 following treatment with NRG, NRG and AGRN and AGRN over a 24-hour time course (A). Expression levels of Egr3 were quantified and are represented in B and C. NRG appeared to induce Egr3 at all time points and reached a peak by 3 hours, which is earlier than that of AGRN alone at 6 hours (B and C). Addition of NRG and AGRN together appeared to be possibly additive, and a peak in protein level was reached between 3 and 6 hours and remained high for the duration of the 24-hour test (B and C). Although not significant, likely due to the great deal of variation, the implied trend suggests NRG and AGRN are sufficient to induce expression of Egr3 in $\mathrm{C} 2 \mathrm{C} 12$ myotubes (mean $\pm \mathrm{SD} ; \mathrm{n}=3$ ). 

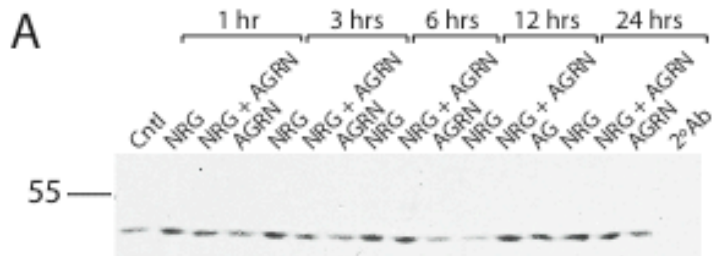

$43-$

B
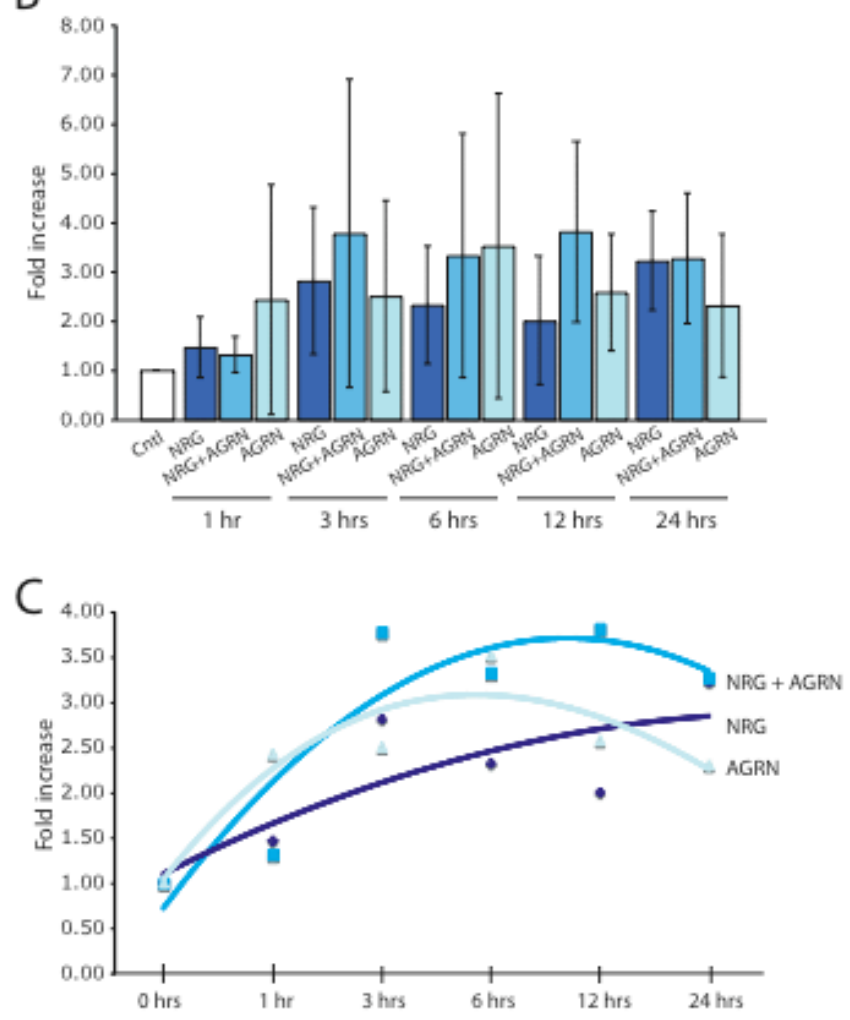
Treatment with AGRN alone appeared to peak at 6 hours, while AGRN and NRG together appeared to peak at some time point between 3 and 6 hours (Figure 3). There were no significant changes (tested by $t$-test) in Egr3 as a consequence of the treatments, likely due to the sizeable amount of variation. The implied trend however, did allow us to determine that 4 hours is an appropriate treatment length for the rest of the study.

To address whether LN was also capable of increasing the induction of Erg3, C2C12 myotubes were treated for 4 hours with NRG, NRG $+\mathrm{LN}$ and $\mathrm{LN}$ alone. The resulting western blot analysis revealed that the addition of $\mathrm{LN}$ results in a roughly $100 \%$ increase in protein level over the basal level of the untreated group ( $\mathrm{n}=3, P<0.01, t$-test; Figure 4). Addition of NRG increased Egr3 by $\sim 50 \%$ in the same experiment ( $\mathrm{n}=3$, $P<0.05, t$-test), and NRG and LN together resulted in a $\sim 75 \%$ increase ( $\mathrm{n}=3, P<0.05, t$ test). Therefore, $\mathrm{LN}$ is able to increase the expression of Egr3, but treatment with NRG and LN simultaneously does not appear to be additive. Interestingly, this data also suggests that under these conditions in the $\mathrm{C} 2 \mathrm{C} 12$ culture, $\mathrm{LN}$ is sufficient to induce Egr3. 


\section{Figure 4: LN induced Egr3 expression in C2C12 myotubes}

Western blot for Egr3 after 4 hour incubation with NRG, NRG and LN and LN alone (A). Egr3

protein levels were determined using densitometry and are represented as averages (mean \pm SD)

in B. Addition of NRG increased Egr3 by $50 \%(n=3, * P<0.05)$ and NRG and $\mathrm{LN}$ together increased Egr3 levels by $75 \%(\mathrm{n}=3, * P<0.05)$. $\mathrm{LN}$ addition resulted in a $100 \%$ increase of Egr3 compared to the basal level of Egr3 in the untreated control $(\mathrm{n}=3, * * P<0.01)$. The addition of NRG and LN together does not appear to be additive according to this data. 
A
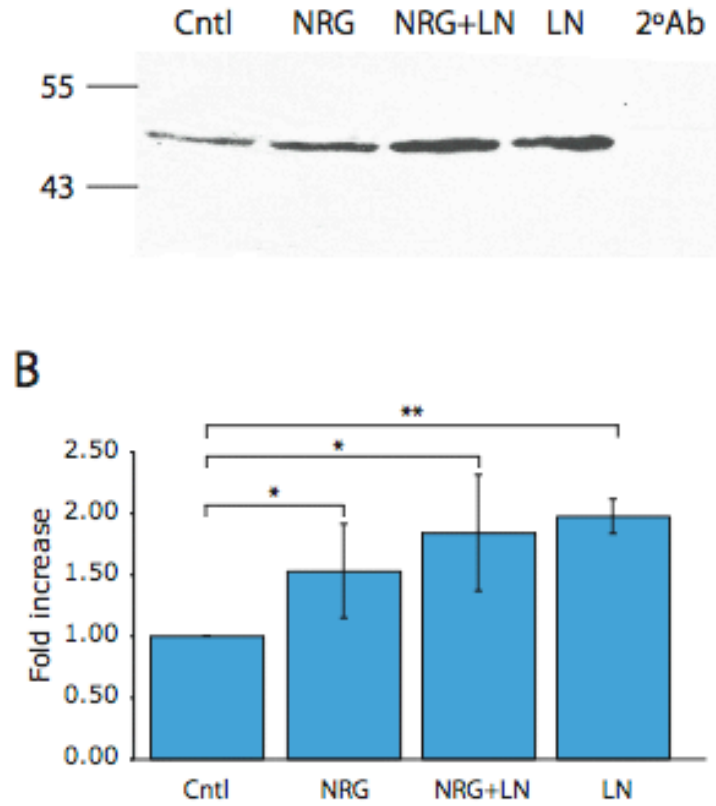


\section{2 $\alpha-D G$ is necessary for NRG, ARGN and LN induced Egr3 expression}

Since both AGRN and LN bind to $\alpha$-DG (Bowe et al., 1994; Campanelli et al., 1994; Gee et al., 1994; Ibraghimov-Beskrovnaya et al., 1992; Sugiyama et al., 1994) and it was shown that both AGRN and LN induce Egr3, it was relevant to determine whether this induction is diminished if $\alpha$-DG is extensively reduced. In order to address this, the $\alpha$-DG-silenced myotube cell line, 11E, was used. 11E myotubes were generated from C2C12 cultures into which a stable $\alpha-D G$ anti-sense construct has been integrated (Montanaro et al., 1999). The resulting cell line exhibits an $\sim 80 \%$ reduction in $\alpha$-DG protein (Jacobson et al., 1998). Upon receiving the 11E cultures, a western blot was performed to ensure the $\alpha$-DG was indeed reduced (Figure 5). In fact there is a $>80 \%$ decrease in $\alpha-\mathrm{DG}$ in $11 \mathrm{E}$ cultures compared to $\mathrm{C} 2 \mathrm{C} 12$ cultures (Figure 5).

Following confirmation of $\alpha-\mathrm{DG}$ reduction in 11E cultures, it was necessary to establish whether the increases in Egr3 attributed to NRG and ARGN were affected by decreased $\alpha$-DG. C2C12 and 11E cultures were both treated with NRG, NRG + AGRN and AGRN for 4 hours. A noticeable decline in Egr3 in treated 11E compared to $\mathrm{C} 2 \mathrm{C} 12$ cultures is shown in a representative western blot (Figure 6A). There was a $79.7 \pm 4.7 \%$ $($ mean $\pm \mathrm{SD} ; \mathrm{n}=3, P<0.01, t$-test) reduction in NRG-induced Egr3 and an $80.4 \pm 8.9 \%$ $(\mathrm{n}=3, P<0.05, t$-test) reduction in NRG and AGRN-induced Egr3 production in $11 \mathrm{E}$ treated groups (Figure 6B). AGRN stimulated 11E cultures also produced less Egr3 than their $\mathrm{C} 2 \mathrm{C} 12$ counterparts (reduced by $72.0 \pm 13.3 \%$; Figure $6 \mathrm{~B}$ ).

In order to investigate whether the diminished Erg3 induction exhibited by NRG and AGRN treated 11E cells was also observed in LN treated 11E cultures, a similar 


\section{Figure 5: Confirmation of $\alpha-D G$ reduction in $11 \mathrm{E}$ myotubes}

Western blot for $\alpha-\mathrm{DG}$ in $\mathrm{C} 2 \mathrm{C} 12$ and 11E myotubes (A). The amount of $\alpha$-DG protein present in $\mathrm{C} 2 \mathrm{C} 12$ and $11 \mathrm{E}$ myotubes was quantified and expressed as a percentage of the amount of $\alpha$-DG present in $\mathrm{C} 2 \mathrm{C} 12$ myotubes (B). There is an approximate $80 \%$

reduction in the amount of $\alpha-\mathrm{DG}$ expressed in $11 \mathrm{E}$ myotubes compared to $\mathrm{C} 2 \mathrm{C} 12$ myotubes $(n=1)$. 

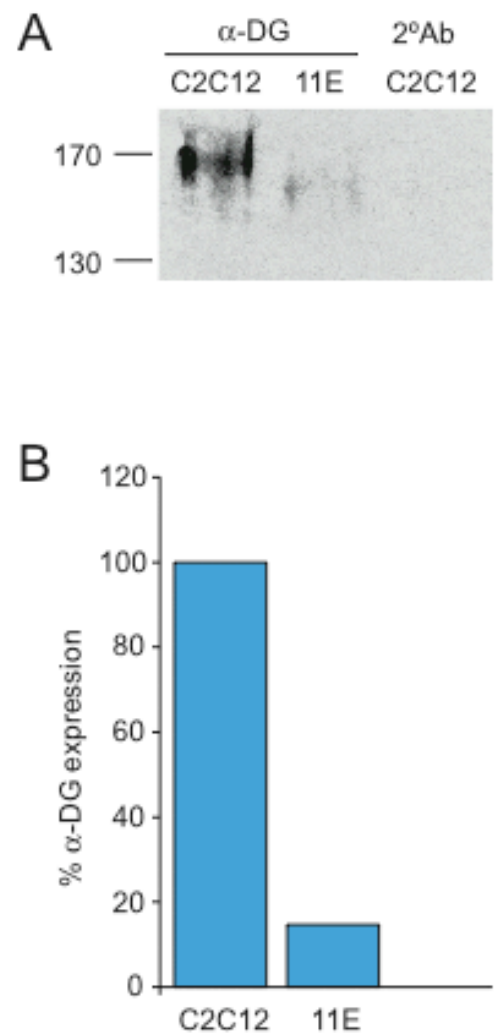
Figure 6: $\alpha-D G$ is required for NRG and AGRN induction of Egr3

Representative immunoblot for Egr3 induced by 4 hour treatments of NRG and NRG and AGRN and AGRN alone in C2C12 and 11E myotubes (A). Levels of Egr3 protein were quantified by densitometry and reported as averages (mean $\pm \mathrm{SD} ; \mathrm{n}=3$ ) in $\mathrm{B}$. In all treatment groups the amount of Egr3 was substantially reduced in the $11 \mathrm{E}$ myotubes when compared to the same treatments in the $\mathrm{C} 2 \mathrm{C} 12$ myotubes (panel $\mathrm{B} ; \mathrm{n}=3, * P<0.05$, **P<0.01). Induction of Egr3 by NRG and AGRN in $11 \mathrm{E}$ myotubes was reduced by 79.7 $\pm 4.7 \%$ and $72.0 \pm 13.1 \%$ respectively. A similar reduction was also observed in $11 \mathrm{E}$ cultures treated with both NRG and AGRN together when compared to the same treatment in $\mathrm{C} 2 \mathrm{C} 12$ myotubes $(80.4 \pm 8.9 \%)$. 

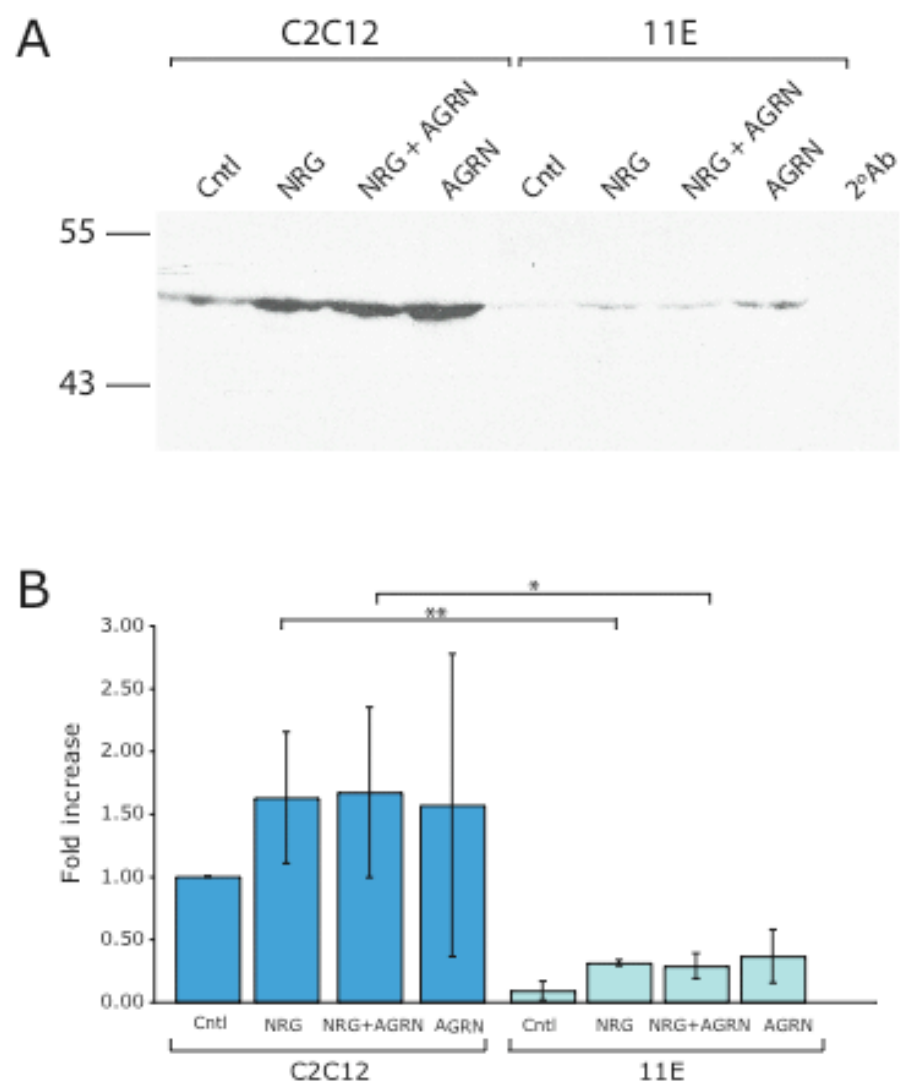
experiment was performed using LN instead of AGRN. Both C2C12 and 11E cultures were treated for 4 hours and protein extracted and immunoblot analysis carried out. Again, all Egr3 induction levels were substantially reduced in 11E treatment groups. There was a $94.0 \pm 2.4 \%(\mathrm{n}=3, P<0.05, t$-test $)$ decrease in NRG-induced Egr3 and a 92.0 $\pm 3.4 \%$ decrease in $\mathrm{LN}$-induced Egr3 production in 11E myotubes compared to that of C2C12 cultures (Figure 7).

To confirm this decrease in NRG and LN induced Egr3 expression is due to reduced levels of $\alpha$-DG in the 11E myotubes, an $\alpha$-DG antibody blocking experiment was executed. $\mathrm{C} 2 \mathrm{C} 12$ cultures were treated with a 1:50 dilution of the $\alpha$-DG blocking antibody $11 \mathrm{H} 6$ for 1 hour prior to the addition of 4-hour treatments of NRG and LN (as well as an untreated control). A representative immunoblot illustrates the reduced NRGand LN-induced Egr3 levels when $\alpha$-DG is obstructed (Figure 8A). Although densitometric analysis outcome yielded results with no significance $(n=7)$ between treatment groups and the treatment with the $\alpha$-DG function-blocking antibody, the implied trend suggests that blocking $\alpha$-DG does actually diminish Egr3 induction (Figure $8 \mathrm{~B})$. Taken together along with the $\mathrm{C} 2 \mathrm{C} 12$ vs. $11 \mathrm{E}$ experiments, this data suggests that NRG, AGRN and LN induction of Egr3 is either directly or indirectly mediated by $\alpha-$ DG.

Since Egr3 expression appears to be mediated by $\alpha-D G$, whether this also translated into a reduction of sd-MyHC, a specific $\mathrm{MyHC}$ variant unique to muscle spindle fibers was investigated. Over night treatment of $\mathrm{C} 2 \mathrm{C} 12$ and $11 \mathrm{E}$ cultures with NRG and LN were fixed in ice-cold ethanol and used for indirect immunofluorescence for sd-MyHC (Figure 9). Qualitative examination of the sd-MyHC specific fluorescence 
revealed only very minor, inconsequential changes in fluorescence between $\mathrm{C} 2 \mathrm{C} 12$ and $11 \mathrm{E}$ myotubes for each treatment group. Unexpectedly, there was no discernible increase in sd-MyHC in the $\mathrm{C} 2 \mathrm{C} 12 \mathrm{NRG}$ treated myotubes compared to the untreated control group. This was unanticipated as a previous group conducted a similar experiment with comparable treatment conditions in a primary human myotube cell line, which yielded substantial increases in sd-MyHC following NRG treatment (Jacobson et al., 2004). Perhaps this can be attributed to the fact that $\mathrm{C} 2 \mathrm{C} 12$ myotubes were originally generated from adult mice instead of embryonic mice and have somehow lost the ability to be persuaded to produce sd-MyHC (Blau et al., 1983). 
Figure 7: $\alpha-D G$ is required for NRG and LN induction of Egr3

Western blot of 4 hour NRG, NRG and LN and LN treated C2C12 and 11E myotubes with an antibody specific to Egr3 (A). Quantified Egr3 protein levels expressed as averages (mean $\pm \mathrm{SD} ; \mathrm{n}=3$ ) are shown in $\mathrm{B}$. The amount of Egr3 protein produced was significantly reduced in $11 \mathrm{E}$ myotubes, which have substantially reduced amounts of $\alpha-$ DG, when compared to $\mathrm{C} 2 \mathrm{C} 12$ myotubes $(\mathrm{B} ; \mathrm{n}=3, * P<0.05)$. NRG induction was reduced by $94.0 \pm 2.4 \%$ and $\mathrm{LN}$ induction of Egr3 by $92.0 \pm 3.3 \%$ in $11 \mathrm{E}$ cultures. The induction of Egr3 observed in C2C12 cultures was not observed in the 11E myotubes (B). 
A

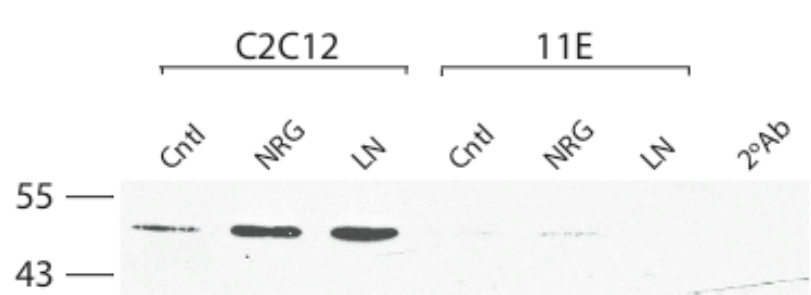

B

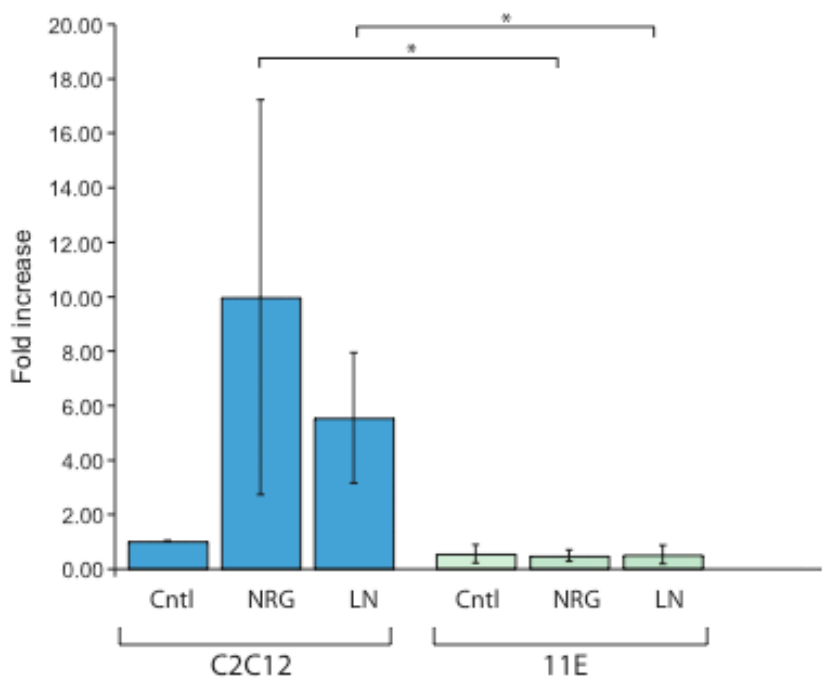




\section{Figure 8: Blocking $\alpha$-DG reduced the induction of Egr3}

Western blot of blocking NRG- and LN-induced Egr3 expression using the monoclonal antibody $11 \mathrm{H} 6$, which blocks $\alpha$-DG (A). Densitometry was analyzed and reported as mean $\pm \operatorname{SD}(n=7)$ in panel B. Although not significant, most likely due to the high levels of variability, the implied trend suggests that by blocking $\alpha$-DG the amount of Egr3 induced by either NRG or LN treatment is reduced (B). 
A

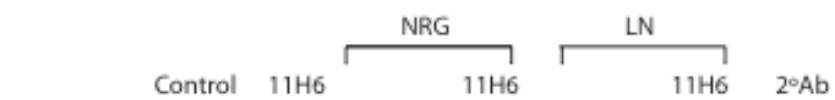

$55-$

11H6 20Ab

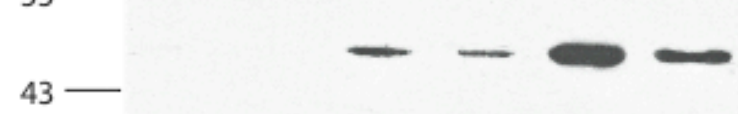

B

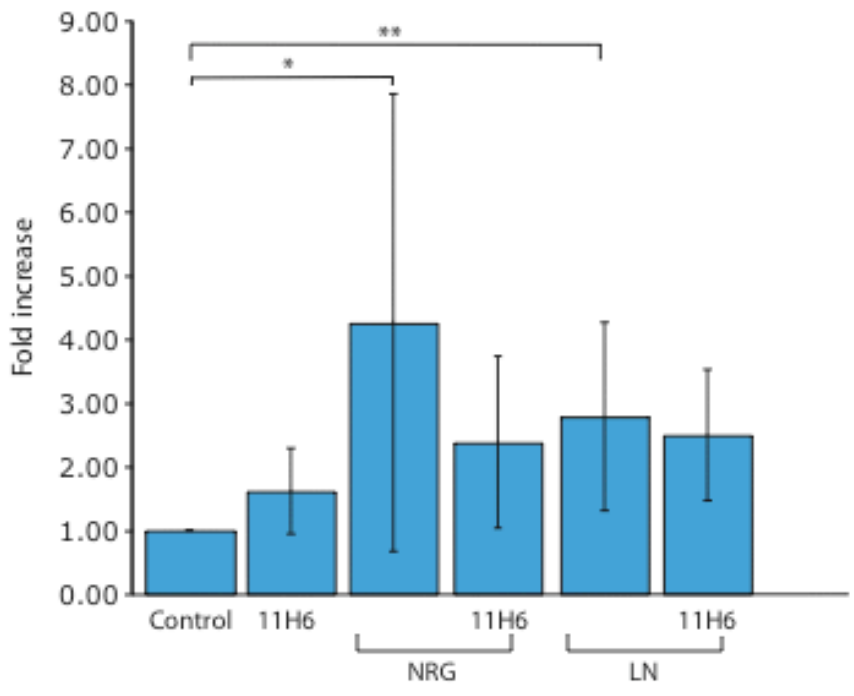




\section{Figure 9: The effect of reduced $\alpha-D G$ on sd-MyHC}

C2C12 and 11E myotubes were left untreated and treated with NRG and LN for 18 hour and subsequently fixed in ice-cold ethanol. Immunocytochemistry was used to visualize changes in sd-MyHC in the different treatment groups. There was no observable changes in sd-MyHC specific fluorescence between $\mathrm{C} 2 \mathrm{C} 12$ and $11 \mathrm{E}$ treated myotubes. Even the C2C12 myotubes showed little evidence of increased sd-MyHC in the NRG treated group compared to the background levels of non-specific fluorescence in the secondary antibody control panel. Scale bar, $25 \mu \mathrm{m}$ for $\mathrm{C} 2 \mathrm{C} 12$ and $11 \mathrm{E}$ treated myotubes and 90 $\mu \mathrm{m}$ in the secondary antibody control. 

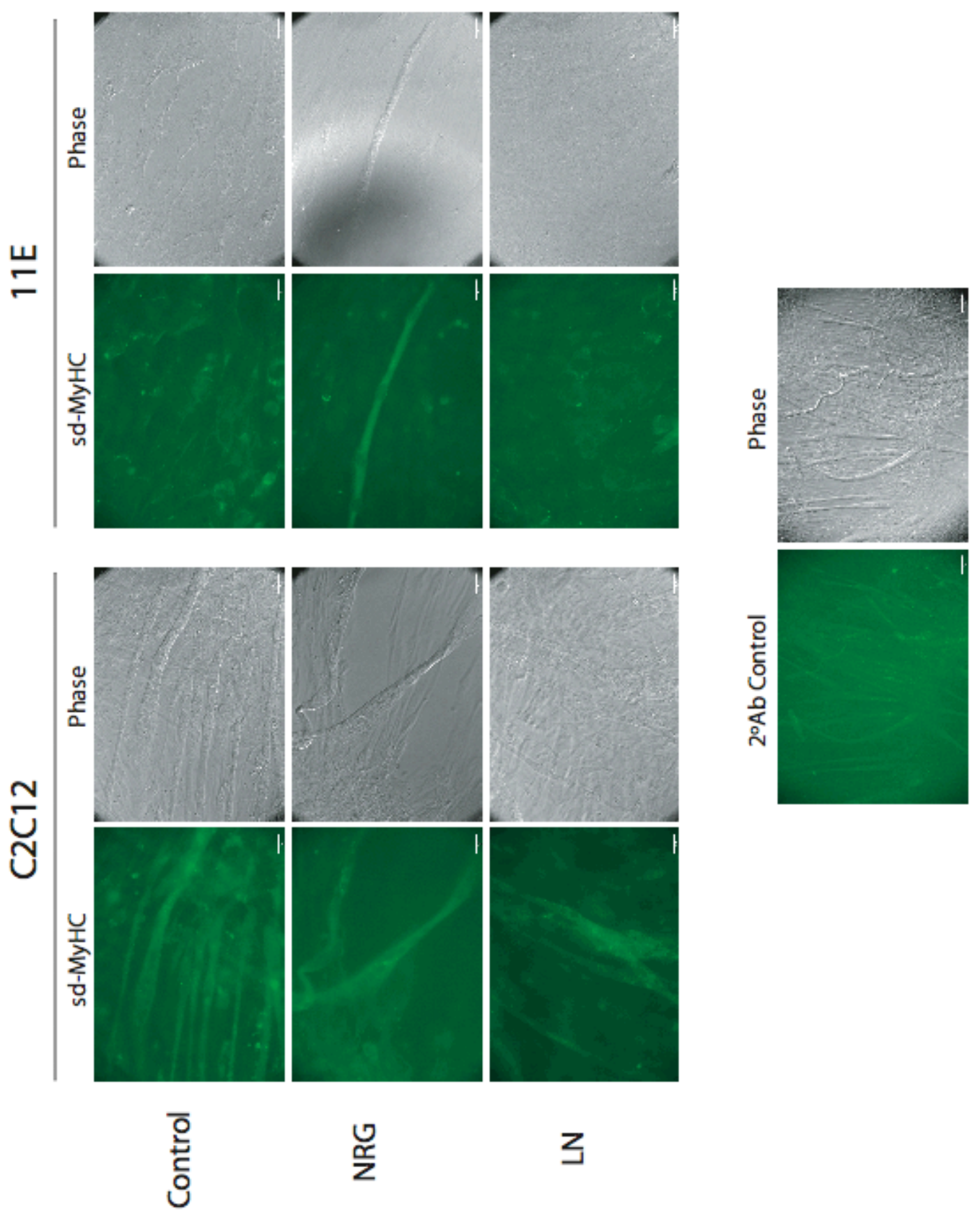


\subsection{LN-stimulated Egr3 induction is reduced by NRG and ErbB inhibitors}

It was observed that NRG and LN induction of Egr3 is disrupted by a decrease in $\alpha$-DG levels and by blocking $\alpha$-DG. Following this, the question of what effect blocking NRG signaling had on NRG and LN-induced Egr3 production was addressed. Firstly, a function-blocking anti-NRG antibody was used to directly block NRG. C2C12 cultures were treated with NRG and LN as well as an untreated control along with the addition of $2 \mu \mathrm{g} / \mathrm{ml}$ of anti-NRG1- $\beta 1$ EGF domain-neutralizing antibody for 2 hours. The subsequent levels of Egr3 were monitored by western blot (Figure 10). Quantified Egr3 levels reveal an increase of $\sim 3.5$ and $\sim 2.5$ fold in NRG and LN treated myotubes respectively, compared to the untreated control (Figure 10B; $=3, P<0.05, P<0.001$, $t$-test). These heightened Egr3 levels were decreased by $36.6 \pm 16.6 \%$ and $33.0 \pm 15.9 \% \quad(n=3, P<0.05$, $t$-test) with the addition of the NRG-blocking antibody (Figure 10).

Secondly, the ErbB receptor inhibitor Tyrphostin AG825 was used to block ErbB signaling into the cell. Tyrphostin AG825 is an adenosine triphosphate (ATP) competitive inhibitor that specifically competes with ErbB receptors for ATP, which is necessary for its tyrosine kinase activity. As with the NRG-blocking experiments, $\mathrm{C} 2 \mathrm{C} 12$ myotubes were treated with NRG, LN and an untreated control for 4 hours prior to protein extraction. The AG825 was added to cultures overnight before the addition of treatments. Immunoblot analysis was used to examine changes in Egr3 expression (Figure 11). Densitometry was used to quantify values and all were expressed as a percentage of the untreated control, which was standardized to zero to allow many independent experiments to be compared. A representative western blot illustrates the 
observable reduction in Egr3 induction by the ErbB receptor signaling inhibitor AG825 (Figure 11A). Addition of AG825 caused a reduction in NRG and LN stimulated Egr3 induction by $61.2 \pm 23.1 \%(\mathrm{n}=3, P<0.01, t$-test $)$ and $51.6 \pm 26.4 \%(\mathrm{n}=3, P<0.01, t$-test $)$ respectively (Figure 11B). Taken together, this data suggests that the increase in Egr3 produced by the addition of $\mathrm{LN}$ is mediated through the NRG-ErbB, ligand-receptor complex in NRG independent manner and not another alternative pathway. 


\section{Figure 10: Blocking NRG reduces LN-induced Egr3 expression}

Imunoblot of NRG- and LN-induced Egr3 expression reduction using a function blocking monoclonal antibody specific to NRG (A). Quantified protein levels were expressed as averages (mean $\pm \mathrm{SD}, \mathrm{n}=3$ ) shown in $\mathrm{B}$. NRG and $\mathrm{LN}$ induction compared to the untreated control was increased by $\sim 3.5$ and $\sim 2.5$ fold respectively $(n=3, * P<0.05$, $* * * P<0.001)$. This increase in Egr3 production induced by NRG was reduced by $36.6 \pm$ $16.6 \%$ in the presence of the NRG-blocking antibody $(n=3)$. Likewise, the LN-induced Egr3 expression was also reduced with the addition of the blocking antibody by $33.0 \pm$ $15.9 \%(\mathrm{n}=3, * P<0.05)$. 
A

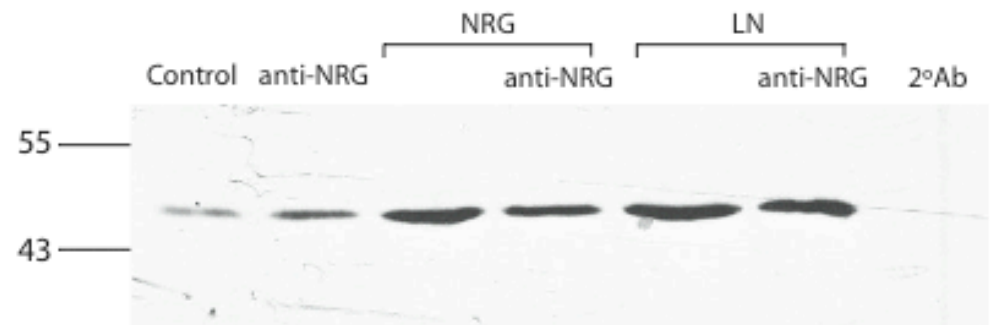

B

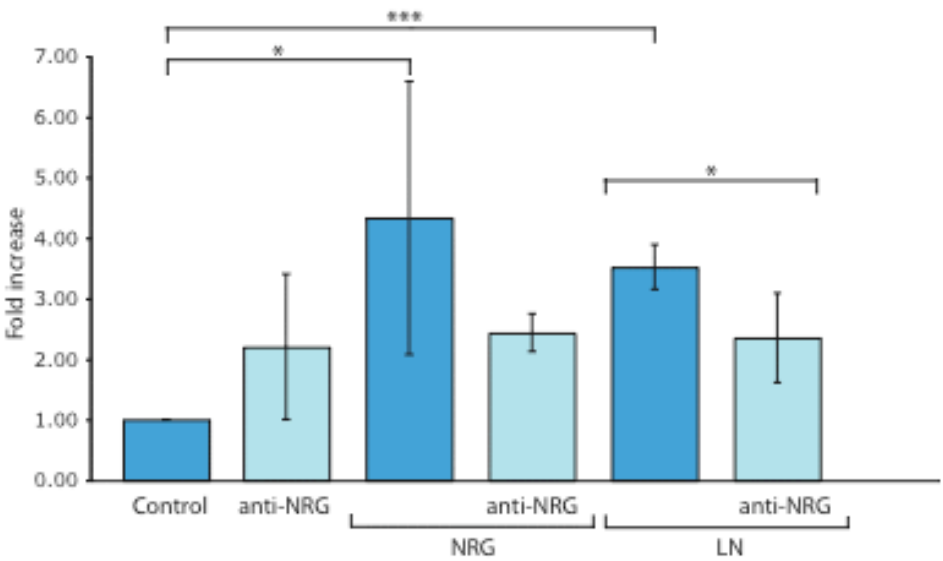


Figure 11: Blocking ErbB receptor signaling reduces LN-induced Egr3 expression Panel A illustrates a Western blot demonstrating the reduced NRG and LN induction of Egr3 expression using the ErbB receptor inhibitor AG825. Quantified protein levels were expressed as averages (mean $\pm \mathrm{SD}, \mathrm{n}=3$ ) shown in B. Blocking ErbB signaling resulted in a reduction of NRG-induced Egr3 expression by $61.2 \pm 23.1 \%(\mathrm{n}=3, * * P<0.01)$ and a reduction in $\mathrm{LN}$-induced Egr3 expression by $54.6 \pm 26.4 \% \quad(\mathrm{n}=3, * P<0.05)$. 

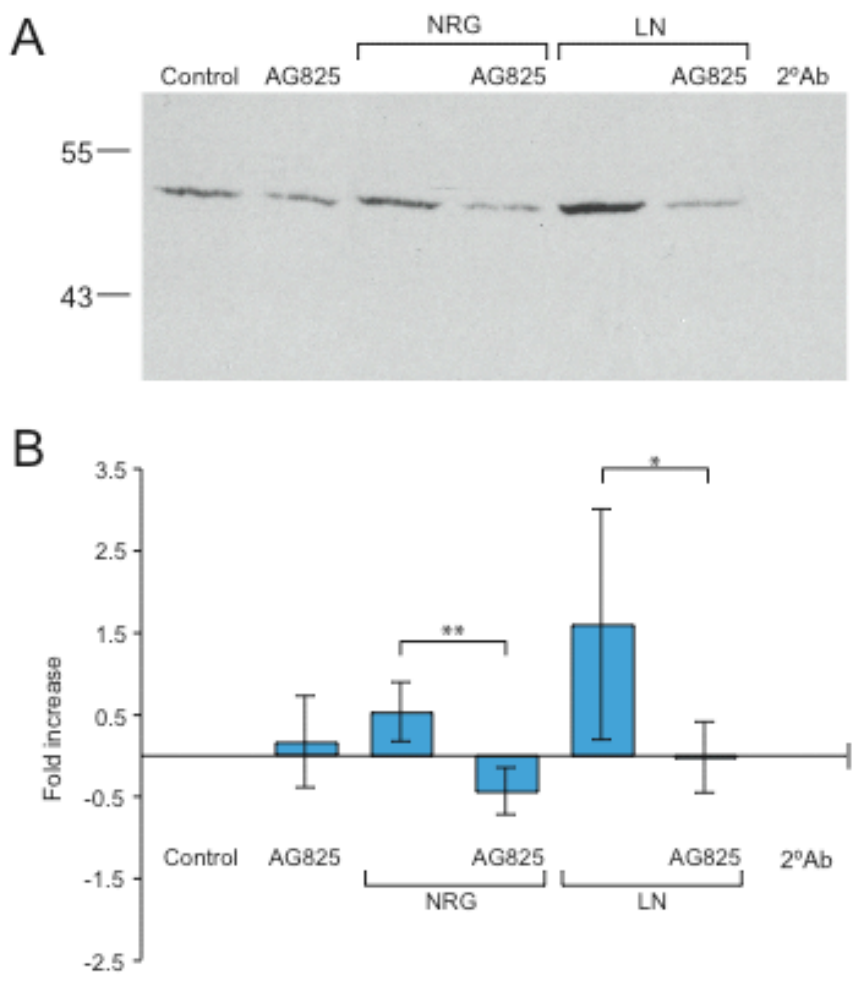


\subsection{Involvement of $\alpha-D G$ in ErbB receptor clustering}

These results show that LN and AGRN, both $\alpha$-DG ligands, are sufficient for induction of Egr3. The results also show that $\alpha$-DG is essential for both LN and AGRN induction of Egr3 as well as NRG induced expression of Egr3. Both LN and AGRN act to cluster AChRs at the NMJ and ErbB receptors colocalize with AGRN-induced AChR aggregates (Gautam et al., 1996; Meier et al., 1997; Rimer et al., 1998). Given this, it was then attempted to determine whether $\alpha-D G$ is obligatory in this co-clustering process.

$\mathrm{C} 2 \mathrm{C} 12$ and 11E myotubes were incubated with NRG and LN (and an untreated control) overnight and AChRs were then directly labeled by adding rhodamineconjugated $\alpha$-BTX to the cultures prior to being fixed in paraformaldehyde. Following fixation, ErbB3 receptors were indirectly stained with anti-ErbB3 specific antibodies followed by incubation with a fluorescence-conjugated antisera. Slides were examined and photographed at a final magnification of $400 \times$. From these photographs, AChR and overlapping ErbB3 clusters were quantified and the ratio of number of $\mathrm{AChR}$ clusters per myotube was calculated (Table 1). In $\mathrm{C} 2 \mathrm{C} 12$ myotubes, the addition of NRG compared to the untreated control showed little change in the number of AChR clusters per myotube and about half of the AChR clusters had overlapping ErbB3 aggregates in both the NRG treated and control (Figure 12A, arrows). The untreated and NRG treated myotubes had similar number of AChR clusters per myotube, 1.15 and 1.11 respectively (Table 1). In contrast, there was a substantial increase in the amount of AChR clusters in the $\mathrm{LN}$ treated myotubes (Figure 12A, arrows). The average number of AChR clusters

per myotube was calculated to be 2.15 (Table 1). Therefore the addition of $\mathrm{LN}$ to $\mathrm{C} 2 \mathrm{C} 12$ myotubes greatly increases the number of AChR clusters per myotube. 
In the absence of abundant $\alpha$-DG, a drastic reduction in AChR clusters is observed (Figure 12B arrows, Table 1). In all 11E treatment groups the ratio of AChR clusters to number of myotubes is radically decreased. In the $11 \mathrm{E}$ untreated group, the number of AChR cluster per myotube was diminished to 0.52 and in the NRG and LN treated groups similar values of 0.67 and 0.59 were computed (Table 1).

Surprisingly, in all $\mathrm{C} 2 \mathrm{C} 12$ and $11 \mathrm{E}$ treatment groups there was not a considerable amount of variability in the percent of AChR clusters that scored positive for ErbB3 clusters (Figure 12A,B arrow, Table 1). Addition of $\mathrm{LN}$ to $\mathrm{C} 2 \mathrm{C} 12$ myotubes results in an increased number of $\mathrm{AChR}$ receptor aggregates (2.15 AChR clusters per myotube, $\mathrm{n}=241$ ) and $53 \%$ of those had ErbB3 clusters that overlaped (Table 1). Similarly, untreated $\mathrm{C} 2 \mathrm{C} 12$ cultures and $\mathrm{NRG}$ treated cultures exhibit 59\% and $67 \%$ of the $\mathrm{AChR}$ clusters having overlap. The number of AChR clusters is considerably increased in the presence of LN and so is the number of ErbB3 clusters, but the proportion of overlap remains similar to the other two groups where clustering is likely spontaneous. Interestingly, the extent of AChR-ErbB3 overlap without typical levels of $\alpha$-DG remains quite constant at $50 \%$ in the untreated control and $47 \%$ and $59 \%$ in the NRG and LN treated 11E myotubes (Table 1). Taken as a whole, these results show that LN does increase the number of $\mathrm{AChR}$ clusters and approximately half of those are colocalized with ErbB3 clusters. Likewise, in conditions of scarce $\alpha$-DG, about half of the AChR clusters are co-clustered with ErbB3 aggregates. The difference to take notice of, is the increased number of $\mathrm{AChR}$ clusters and therefore the increased number of overlapping ErbB3 aggregates, albeit the proportion remains largely unchanged in the 11E treated myotubes. 


\section{Figure 12: Reduced $\alpha-D G$ decreases the number of $A C h R$ and ErbB3 aggregates}

C2C12 and 11E myotubes were treated with NRG, LN along with an untreated control overnight. Cultures were then fixed and double labeled for AChR clusters and ErbB3 aggregates with rhodamine-labeled $\alpha$-BTX (red channel) and indirect fluorescence with ErbB3-specific antibodies (green channel) respectively. Panel A shows the fluorescence in $\mathrm{C} 2 \mathrm{C} 12$ myotubes. The first column stained for AChR clusters and the middle for ErbB3 clusters. Arrows indicate a cluster in the AChR panel and arrows in the middle column point out areas of increased ErbB3 fluorescence that corresponds to AChR aggregates in the first panel. There is a more considerable amount AChR clusters in cultures treated with LN compared to that of the NRG and control groups (A). The same treatments and staining in $11 \mathrm{E}$ cultures show a remarkable reduction in the number of AChR clusters (B). Labeling controls were also preformed to ensure binding was specific (C). Scale bar, $25 \mu \mathrm{m}$. 


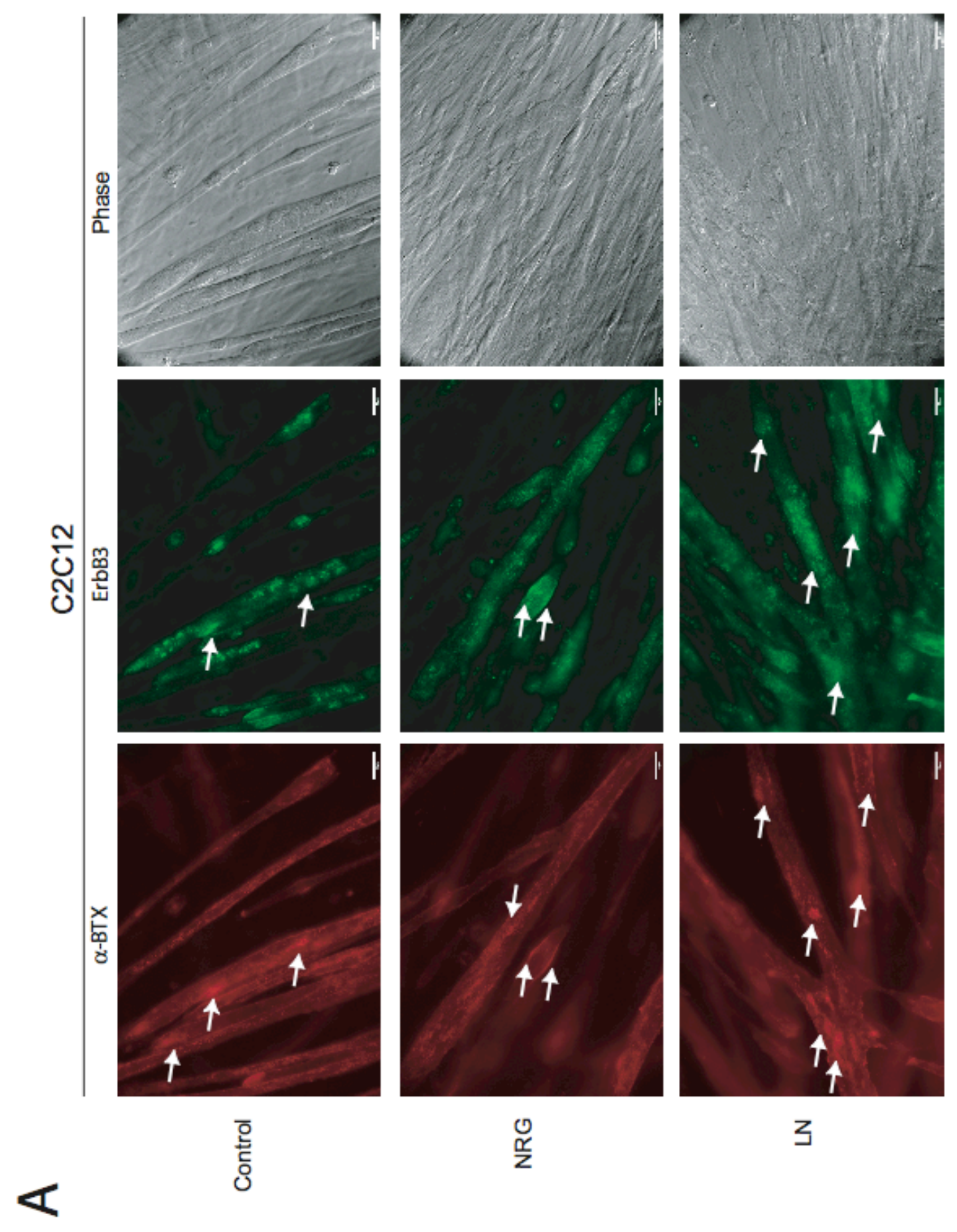




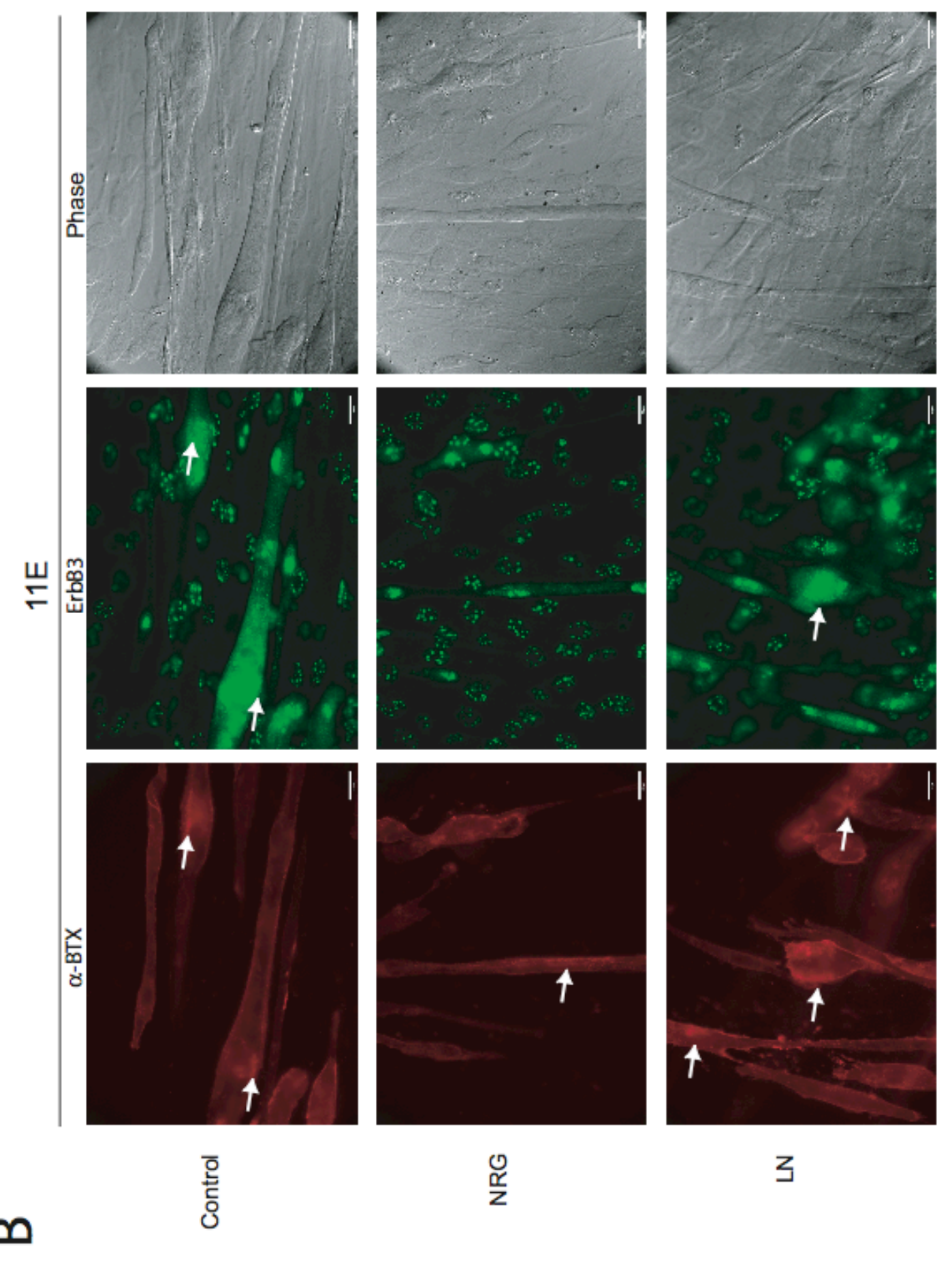



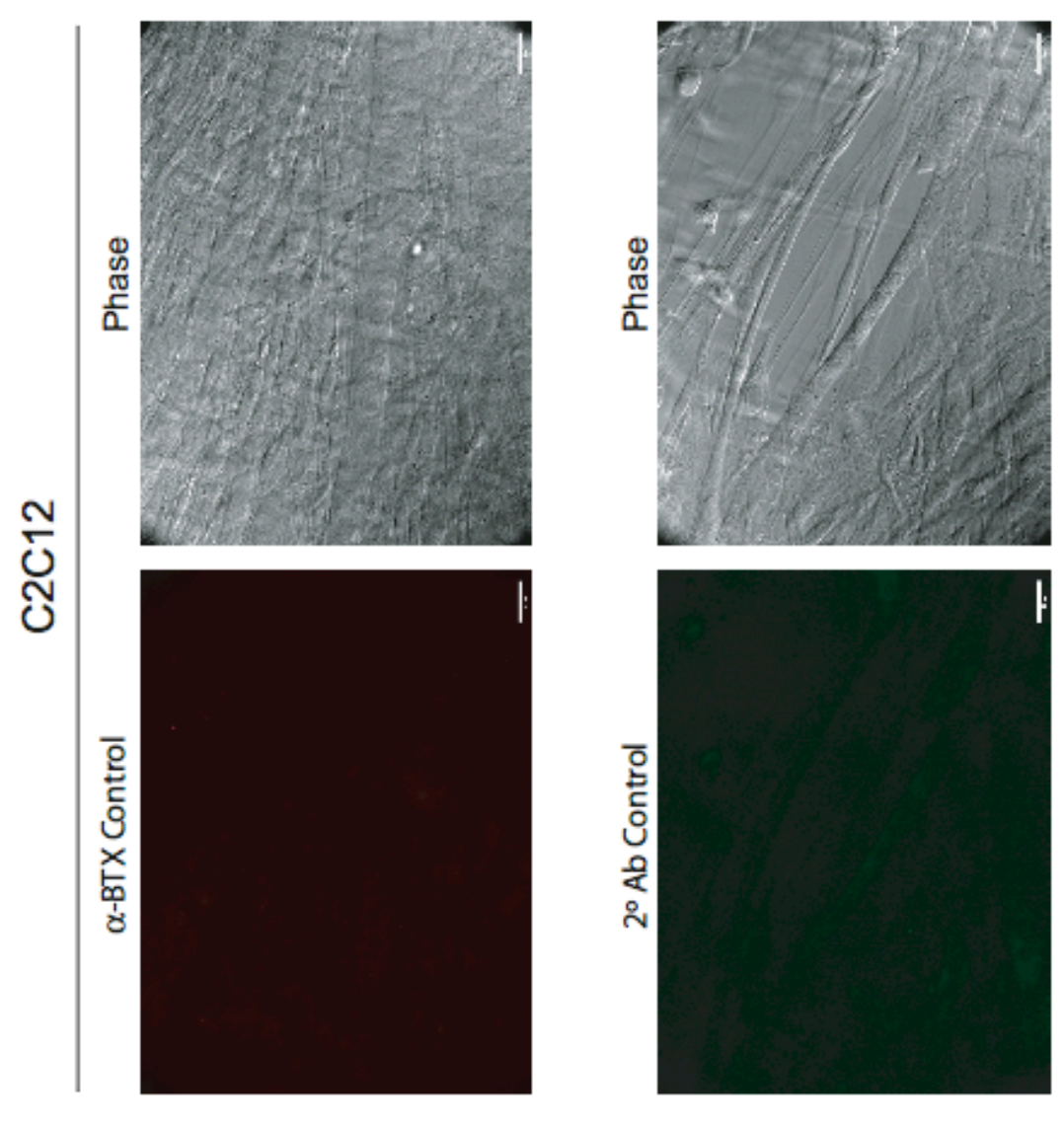


\section{Table 1: Overlap of ErbB3 clusters with AChR aggregates}

C2C12 and 11E myotubes were treated with NRG, LN and an untreated control overnight and were double labeled for AChR clusters and ErbB3 aggregates with rhodaminelabeled $\alpha$-BTX and indirect fluorescence with ErbB3-specific antibodies. Visual inspection was carried out to quantify the extent of ErbB3 overlap with AChR clusters. An overlap was counted if $>50 \%$ of the ErbB3 fluorescence coexisted with that of the AChR cluster fluorescence. The data in the table describes the amount of AChR clusters that scored positive for overlap with ErbB3 aggregates as a percentage with the total number of $\mathrm{AChR}$ clusters counted for the given treatment given in brackets. 


\begin{tabular}{|c|c|c|c|c|}
\hline & \multicolumn{2}{|l|}{$\mathrm{C} 2 \mathrm{C} 12$} & \multicolumn{2}{|l|}{$11 \mathrm{E}$} \\
\hline & AChR clusters/myotube & $\%$ Overlap & AChR clusters/myotube & $\%$ Overlap \\
\hline Control & 1.15 & $59 \% \quad(n=147)$ & 0.52 & $50 \% \quad(n=63)$ \\
\hline NRG & 1.11 & $67 \% \quad(n=184)$ & 0.67 & $47 \% \quad(n=42)$ \\
\hline LN & 2.15 & $53 \% \quad(n=241)$ & 0.59 & $48 \% \quad(n=78)$ \\
\hline
\end{tabular}




\section{Chapter 4}

\section{Discussion}

ECM proteins play a critical role in the development and maintenance of NMJs. Here the possibility of a role for the basal lamina proteins LN, AGRN and their common receptor $\alpha$-DG at sensory synapses in the induction of muscle spindle fibers was investigated. In initial aspects of the study, the induction of the transcription factor Egr3 was used as a marker for early spindle fiber development. It was originally hypothesized that AGRN, LN and $\alpha-\mathrm{DG}$ would be vital elements that act to increase the induction of Egr3, possibly through clustering of the ErbB receptors through which NRG signals. This would be an interesting result as AGRN, LN and $\alpha$-DG all influence important changes at NMJs during development and have not been attributed to similar roles at sensory synapses. Here, it was shown that both AGRN and LN can induce Egr3 expression independent of added NRG. This induction is ablated when ErbB receptor and NRG inhibitors are applied prior to treatment. Further, it was shown that $\alpha$-DG is a necessary component in this process and absence of $\alpha$-DG mitigates Egr3 induction as well as reduced the number of induced and spontaneous AChR and co-clustered ErbB3 aggregates.

\subsection{AGRN and LN can induce Erg3 independently from NRG}

Initial experiments were preformed to determine the affect of AGRN and LN on Egr3 expression in myotubes. Given that both AGRN and LN are capable of clustering AChRs at motor synapses, and that AGRN can also cluster ErbB receptors to motor 
synapses, it was hypothesized that AGRN and LN would increase Egr3 expression (McMahan, 1990; Meier et al., 1997; Rimer et al., 1998; Sugiyama et al., 1997). Initial experiments focused on AGRN and sought to determine if AGRN could influence Egr3 protein levels, as AGRN is a potent inducer of AChR clusters at the NMJ and previous studies demonstrate ErbB2 and ErbB3 clustering in myotubes when treated with AGRN, and persistent ErbB2 and ErbB3 co-localization with AChR clusters in vivo (McMahan, 1990; Meier et al., 1997; Rimer et al., 1998). Furthermore, constitutively active MuSK can also cluster ErbB receptors in the absence of AGRN, and this is sufficient to aggregate ErbB2 to AChR clusters (Jones et al., 1999). Although Ia sensory afferents that innervate muscle spindle fibers do not produce the active neural $\operatorname{AGRN}_{(4,8)}$ necessary to activate MuSK (Stone and Nikolics, 1995), they do produce $\operatorname{AGRN}_{(4,0) \text {, }}$ which is effective in clustering at NMJs a high concentrations (Ferns et al., 1993; Stone and Nikolics, 1995). A time course over 24 hours was used to determine if and at what time point addition of AGRN impacted Egr3 expression the most. Immunoblot analysis revealed that AGRN does indeed increase the amount of Egr3 protein produced when added alone almost to nearly the same extent as the addition of NRG to cultured myotubes (Figure 3). It was also determined that at a time point between 3 and 6 hours AGRN-induced Egr3 expression appeared to peak. Erg3 expression continued to remain high until close to 12 hours (Figure 3). This is consistent with previous studies that have determined that AGRN is effective in inducing AChR microclusters of AChRs within 4 hours of application (Sugiyama et al., 1997). When treatments with NRG, NRG with AGRN and AGRN alone were compared it appeared all three treatments induce Egr3 to 
the same extent as NRG. AGRN is likely not acting in an additive manner and potentially may activate Egr3 through a mechanism similar to NRG.

AGRN stimulates the accumulation of AChRs and ErbB receptors (McMahan, 1990; Meier et al., 1997; Rimer et al., 1998), and can induce Egr3 expression (Figure 3) The next step was to determine if LN, which can also cluster AChRs, also affects the amount of Egr3 produced. Myotubes treated with LN for 4 hours demonstrated increased Egr3 protein levels compared to the untreated control (Figure 4). This increase is similar to the increases induced by NRG (Figure 4). This is an interesting result as LN can increase Egr3 expression, presumably by its ability to cluster cell surface molecules on myotubes not unlike it aggregates AChRs at NMJs independent of MuSK activation (Suigyama et al., 1997; Montanaro et al., 1998). Neural AGRN is tightly regulated and secreted solely by $\alpha$-motor neurons, and is not produced by the sensory neurons that stimulate spindle fiber differentiation (Ma et al., 1995; Reist et al., 1992; Stone and Nikolics, 1995). Accordingly, LN is a more ubiquitously expressed and LN-mediated clustering of ErbB receptors in spindle fiber development, independent of AGRN-MuSK signaling (Montanaro et al., 1998; Sugiyama et al., 1997), is a more probable scenario involved in this process than the AGRN-MuSK pathway. LN clusters AChRs by binding $\alpha-D G$ and self-association of LN-LN short arms (Cohen et al., 1997). If this situation is acting in the LN-induction of Egr3, then it would be assumed that $\alpha$-DG is critical in this process, which was further investigated.

Since NRG is indispensable in the process of muscle spindle differentiation, which is marked by induction of Egr3 (Hippenmeyer et al., 2002), how then is addition of LN and AGRN able to induce Egr3 without the addition of NRG? There are a few 
possible explanations. First, that there is another pathway being activated, which also leads to the Egr3 expression. Second, since ErbB receptors have been shown to localize in plasma membrane lipid rafts where their tyrosine phosphorylation is much higher (Ma et al., 2003; Zhou and Carpenter, 2000), the receptors may self activate due to their close proximity and tyrosine phosphorylation-rich environment. Finally, as speculated by Ruegg and Bixby (1998), MuSK- and $\alpha$-DG-mediated aggregation of proteins at developing NMJs results in accumulation of muscle-derived NRGs to this site creating a NRG sink (Ruegg and Bixby, 1998). This explanation is supported by another study by Meier and colleagues (1998) that confirms AGRN does induce clustering of musclederived NRGs to the NMJ and that muscle-derived NRG exhibits the same type of activity as NRGs produced by neurons (Meier et al., 1998). Using ErbB receptor and NRG specific inhibitors, it was shown that blocking either component results in a reduction of LN-stimulated Egr3 induction (Figures 10 and 11). This result can eliminate the possibility of a novel pathway being activated, as both ErbB and NRG inhibitors lessen LN-induced Egr3 expression. If self-activation of the ErbB receptors occurs in this experimental environment, inhibition of ErbB phosphorylation should inhibit the induction of Egr3. The results attained are consistent with this notion as Egr3 levels were reduced in all treatment groups when AG825 was also added. The scenario put forth by Ruegg and Bixby (1998) appears to be a more probable match to previous publications, but the results attained in this study, may suggest otherwise. If the anti-NRG treatment inhibits binding of NRG, whether endogenous or exogenous in source, to the ErbB receptors and therefore their activation, it would be expected that the inhibition treatment would reduce even basal level Egr3 expression in untreated groups. However, the results 
shown in Figure 10 (lane 2 of western blot) suggest this is not occurring, as treatment with the inhibitor does not reduce the basal Egr3 level of protein, it is actually slightly elevated. The anti-NRG treatment does reduce Egr3 expression induced by both the addition of NRG and LN treatments, but not in the untreated control group. The antiNRG antibody was added at a concentration that was sufficient to inhibit approximately $50 \%$ of the added NRG; perhaps higher concentrations would produce a different result in the untreated control group. Although both experiments were informative, the actual mechanism acting in this process likely requires further investigation as well as determining the probable mechanism acting in vivo, where NRG is produced by both nerve and muscle (Meier et al., 1998).

\section{$4.2 \alpha-D G$ is necessary for induction of Egr3}

Here, it was shown that both AGRN and LN can induce Egr3 (Figures 3 and 4) and both are known to bind to $\alpha$-DG (Bowe et al., 1994; Campanelli et al., 1994; Gee et al., 1994; Ibraghimov-Beskrovnaya et al., 1992; Sugiyama et al., 1994). The $\alpha$-DGsilenced, 11E myotube culture was used to answer the question what affect does the AGRN/LN receptor $\alpha$-DG have on Egr3 expression? 11E myotubes are derived from C2C12 myotubes and express an antisense $\alpha-D G$ gene (Montanaro et al., 1999). $\alpha$-DG is reduced by $80-90 \%$ in $11 \mathrm{E}$ myotubes (Figure 5; (Montanaro et al., 1999). In all cases reduction of $\alpha$-DG mitigated NRG-, AGRN- and LN-stimulated Egr3 induction (Figures 6 and 7). This suggests that the induction of Egr3 by both AGRN and LN as well as NRG occurs via an $\alpha$-DG dependent mechanism as opposed to a MuSK dependent mechanism, which is consistent with a previous study (Montanaro et al., 1998). Clusters of AChRs at 
developing NMJs are still present in $\alpha$-DG deficient muscles (Cote et al., 1999; Grady et al., 2000; Jacobson et al., 2001). If the AGRN induced Egr3 were the result of AGRNMuSK signaling, it would be expected that in $\alpha$-DG deficient myotubes that the induction of Egr3 would not be impaired in the absence of $\alpha$-DG. Previous studies have reported that AGRN-MuSK signaling is not substantially inhibited in muscle cell lines deficient in $\alpha$-DG (Jacobson et al., 1998), reinforcing the notion that induction of Egr3 by AGRN occurs independently of MuSK. To determine if the AGRN-MuSK pathway does play a role in spindle fiber differentiation, future studies could investigate whether or not $\mathrm{AGRN}^{-/-}$and $\mathrm{MuSK}^{-/-}$mice develop muscle spindle fibers. As well as investigating the newly identified elements necessary in this process such as Lrp4, Tid1 and Dok-7 and whether these knockout mice have fully formed muscle spindle fibers.

In all treatment groups, the NRG-, LN- and AGRN-induced Egr3 expression was drastically reduced when $\alpha$-DG levels were diminished (Figures 6 and 7). Both LN and AGRN may cluster ErbB receptors, via a diffusion trap type mechanism, as a similar mechanism has been suggested in AChR clustering (Jacobson et al., 2001). Originally, it was thought that AGRN released from innervating neurons initiated postsynaptic differentiation (McMahan, 1990). However, other studies suggest that AGRN may act as a stabilizer or by a de-clustering mechanism rather than an active clustering signal. Prior to motor innervation of skeletal muscle there are spontaneous postsynaptic-like AChR clusters present on the muscle surface (Fischbach and Cohen, 1973). When innervation occurs in $\mathrm{AGRN}^{-/-}$mice, these clusters disperse, but when AGRN is released, it counteracts this dispersal (Lin et al., 2001). LN can also act in a comparable manner by binding $\alpha$-DG and self-assembling into a matrix via its short arms (Figure 1; (Cohen et 
al., 1997). Bezakova and Ruegg (2003) suggest the formation of a LN/integrin-AGRN- $\alpha$ DG network, which could act in a diffusion trap manner (Bezakova and Ruegg, 2003; Cohen et al., 1997). AGRN isoforms without the Z-splice insert bind to $\alpha-D G$ and $L N$ with high affinity (Denzer et al., 1997; Gesemann et al., 1998). This interaction may mediate LN/integrin-AGRN- $\alpha$-DG connections (Burgess et al., 2002). All of these possibilities are consistent with the $\mathrm{C} 2 \mathrm{C} 12$ vs. $11 \mathrm{E}$ experimental results obtained. The exact interactions and mechanisms, including the involvement of integrins, will need to be further investigated to elucidate the actual machinery and mechanisms acting in this process.

To further confirm the importance of $\alpha$-DG in the induction of Egr3, and to ensure the integrity of the myotubes were not jeopardized in creating the $11 \mathrm{E}$ cell line, and that this is not the reason for the reduction in Egr3 induction, the $\alpha$-DG monoclonal antibody was used to block $\alpha$-DG binding to $\mathrm{LN}$. The results show that NRG and LN induced Egr3 expression is reduced when $\alpha$-DG is blocked (Figure 8). This experiment was replicated numerous times and although the level of Egr3 reduction was not significant, a reproducible reduction in Egr3 expression was observed. Previous studies have successfully used this antibody to block $\alpha$-DG-LN interactions (Gee et al., 1994; Montanaro et al., 1998; Montanaro et al., 1999). However, it is important to note that the source of the antibody for these studies was from ascites and was used optimally at a dilution of 1:50 (Gee et al., 1994; Montanaro et al., 1998; Montanaro et al., 1999). The source of the $11 \mathrm{H} 6$ antibody purchased for this study is unknown, but received at a concentration of $200 \mu \mathrm{g} / \mathrm{ml}$, which may be far less than that used in the studies cited (Santa Cruz Biotechnology, Santa Cruz, CA). The antibody was used at a 1:50 dilution, 
as this was used in previous research (Montanaro et al., 1998). A more significant result may have been collected if $11 \mathrm{H} 6$ from ascites were used.

Taken together these results suggest that AGRN and LN induce Egr3 though $\alpha$ DG dependent mechanism. It can be concluded that $\alpha$-DG is an essential element in the induction of Egr3 and therefore likely has an important role in the differentiation of muscle spindle fibers

\section{$4.3 \alpha-D G$ deficiency reduces the number of $A C h R$ and ErbB3}

\section{clusters}

Here, it was shown that addition of $\mathrm{LN}$ to cultured myotubes increases the number of AChR cluster and approximately $50 \%$ of these clusters are co-clustered by ErbB3 (Figure 12 and Table 1). This increase in aggregates is substantially reduced in 11E myotubes lacking $\alpha$-DG (Figure 12 and Table 1). The average number of spontaneous and $\mathrm{LN}$-induced $\mathrm{AChR}$ clusters on $\mathrm{C} 2 \mathrm{C} 12$ myotubes calculated in this study, is consistent with the numbers reported by a previous Montanaro and colleagues (1998). Montanaro and colleagues (1998) also report a significant decrease in AChR aggregates in 11E myotubes, which is not rescued with the addition of $\mathrm{LN}$ this is also consistent with the findings reported here (Montanaro et al., 1998). Interestingly, our finding do demonstrate a marked reduction in AChR clusters in 11E cultures, but the proportion of these aggregates that colocalizes with ErbB3 remains comparably constant (Table 1). This suggests that even in spontaneously formed AChR clusters, approximately $50 \%$ of the clusters will also contain ErbB3 aggregates. An increase in AChR and ErbB3 clustering was observed with $\mathrm{LN}$ treatment and a considerable reduction resulted under $\alpha-\mathrm{DG}$ 
reduced conditions (Figure 12 and Table 1). Under the same conditions, addition of LN and reduction in $\alpha-D G$ resulted in an increase of Egr3 and a reduction of Egr3 respectively. Therefore it is probable that the increase of clustered ErbB3 coincides with an increase in Egr3 expression, and likewise in $\alpha$-DG reduced myotubes a decrease in Egr3.

LN- $\alpha$-DG mediated clustering of ErbB receptors likely results from similar mechanisms acting in clustering AChRs at NMJs. These mechanisms have already been outlined. Another system that parallels this LN- $\alpha-D G$ clustering is the clustering of potassium and water-permeable channels in astrocytes, which are important in potassium buffering and water homeostasis (Guadagno and Moukhles, 2004). Astrocytes are the glial cells of the central nervous system (CNS). Astrocytes grown on a LN substrate showed a substantial increase in the number of AQP4 water-permeable channels and Kir4.1 potassium channels compared to control groups. Furthermore, the potassium channel Kir4.1 was shown to colocalized with the DGC protein syntrophin (Guadagno and Moukhles, 2004; Moukhles and Carbonetto, 2001). This is interesting considering the adaptor protein syntrophin has been shown to associate with ErbB4 localized at NMJs (Garcia et al., 2000). Perhaps interactions with syntrophin are how ErbB receptors are brought into aggregates at NMJs.

\subsection{Conclusions}

So the question remains, is $\alpha$-DG required for muscle spindle differentiation? These results suggest that it may be. Here, it was shown that the basal lamina proteins AGRN and LN are sufficient to induce Egr3, a molecular marker for muscle spindle differentiation, in $\mathrm{C} 2 \mathrm{C} 12$ myotubes just as NRG is capable of doing (Figures 3 and 4). 
LN and AGRN directly associate with $\alpha-\mathrm{DG}$, and limited supplies of $\alpha$-DG resulted in substantially reduced induction of Egr3 (Figures 6,7 and 8). This LN induction appears to be mediated through the NRG-ErbB signaling pathway, as this LN-stimulated increase in Egr3 is diminished by inhibitors of NRG and ErbB receptor signaling (Figures 10 and 11). Furthermore, $\mathrm{LN}$ increases the number of $\mathrm{AChR}$ cluster per myotube with half of them scoring positive for ErbB3 overlap (Table 1). However, this increase is diminished when $\alpha$-DG is reduced (Table 1). Also of interest, although reduced in numbers the same proportion of AChR and ErbB3 clusters overlap (Table 1). Overall these results imply a role for $\alpha-\mathrm{DG}$ and its ligands, AGRN and LN, in the induction of the transcription factor Egr3, which is essential for muscle spindle fiber initiation (Tourtellotte and Milbrandt, 1998). Therefore AGRN, LN and $\alpha$-DG may play a central role upstream of NRG-ErbB signaling in muscle spindle differentiation. Complimentary in vivo studies may assist in clarifying their importance and the mechanisms acting in this process.

\subsection{Future directions}

As mentioned previously, to ultimately determine whether AGRN is involved in differentiation of muscle spindles, it would be interesting to closely examine skeletal muscle sections of AGRN null mice to establish whether or not muscle spindle fibers are present and if there is any variation in the morphology of the spindles compared to their wild type counterparts. AGRN null mice have very few, small, dispersed AChR formations at postsynaptic regions (Gautam et al., 1996). It would also be of interest to determine whether or not MuSK and rapsyn null mice have typical spindle fibers, which both, like the AGRN null mutants, do not have properly formed NMJs (DeChiara et al., 1996; Gautam et al., 1999). Knocking out $\alpha$-DG is an early embryonic lethal mutation 
due to its importance in basement membrane formation, death occurs in development before the advent of spindle differentiation (Williamson et al., 1997). However, chimeric mice, which do have skeletal muscle lacking $\alpha-D G$, could be examined for the presence of typical muscle spindles present in the $\alpha-\mathrm{DG}^{-/-}$muscle to determine the result of $\alpha-\mathrm{DG}$ deficiencies on muscle spindles in vivo (Cote et al., 1999). Such a study would ultimately determine whether $\alpha-D G$ is essential in this process.

Recently the AGRN coreceptor Lrp4 and new proteins involved in AGRN-MuSK signaling have been identified, Tid1 and Dok-7, it would be worthwhile to explore whether these molecules are necessary for spindle formation. More simple experiments that could be carried out to better understand the involvement of AGRN in this process

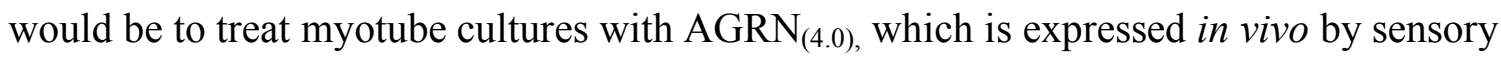
neurons. Since AGRN and LN work together in clustering AChRs at postsynaptic densities, the effect of both LN and AGRN together on Egr3 induction should be examined. A clearer understanding of the mechanisms underlying AGRN and LN clustering of ErbB receptors and subsequent induction of Egr3 will allow a better understanding of how aggregating of proteins works in other systems, such as astrocytes in the CNS, as well as what breaks down and malfunctions in this process in regard to disease states and the aging process. Age-associated degradation of muscle spindle fibers can leave the elderly with proprioceptive deficits that can lead to serious injuries due to falls (Kararizou et al., 2005; Swash and Fox, 1972). Likewise, some diabetic patients are also at increase risk for falls due to altered gait, and decreases in posture control as a result of large-fiber neuropathies associated with abnormal innervation of muscle spindles by Ia afferents (Cavanagh et al., 1992; Muller et al., 2008; Richardson et al., 
2001). Conceivably, better understanding of the mechanisms involved in developing typical muscle spindles, may eventually uncover therapeutic approaches in treating atypical conditions. 


\section{Appendix A}

\section{Figure A1: Western blot of sd-MyHC hybridoma medium}

Western blot of diluted hybridoma medium. Blots were probed with anti-mouse IgG antibodies. The primary antibody mouse-anti-Disc Large was used as a positive control and the IgG fragments are visible below the 55 and $26 \mathrm{kDa}$ markers. The 1:2 dilution of hybridoma mediums is the highest dilution where the IgG light chain is still present and was used as a starting concentration in experiments using the sd-MyHC antibody. 


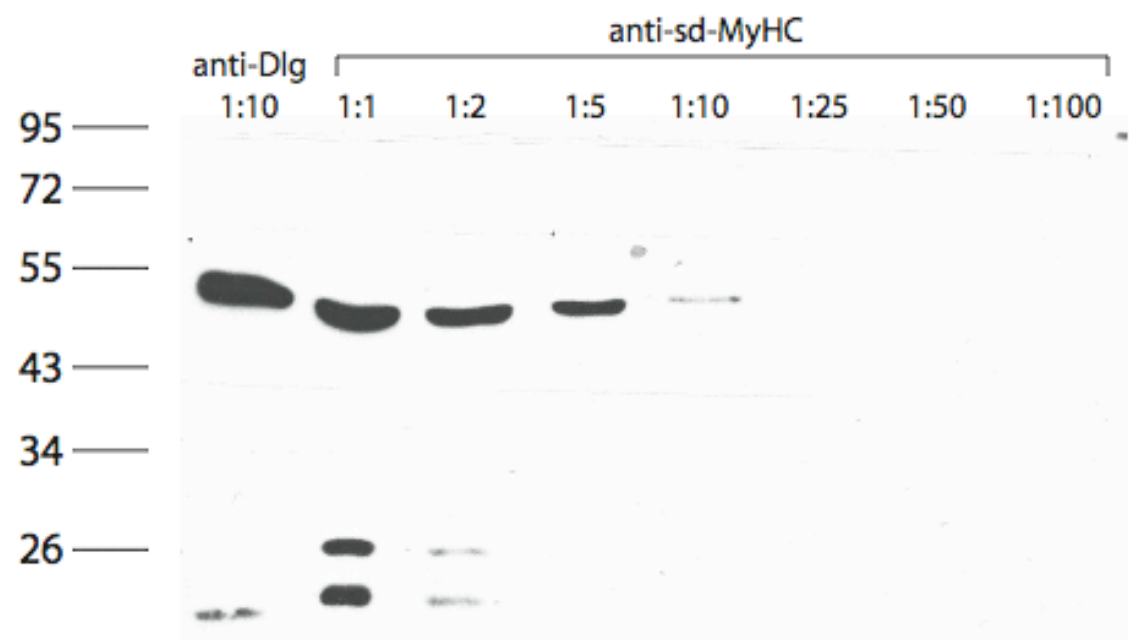


Table A1: AChR/ErbB3 cluster counts and calculations

This table contains the raw data used to calculate AChR clusters/myotube, \% overlap and count the total number of AChR clusters. 


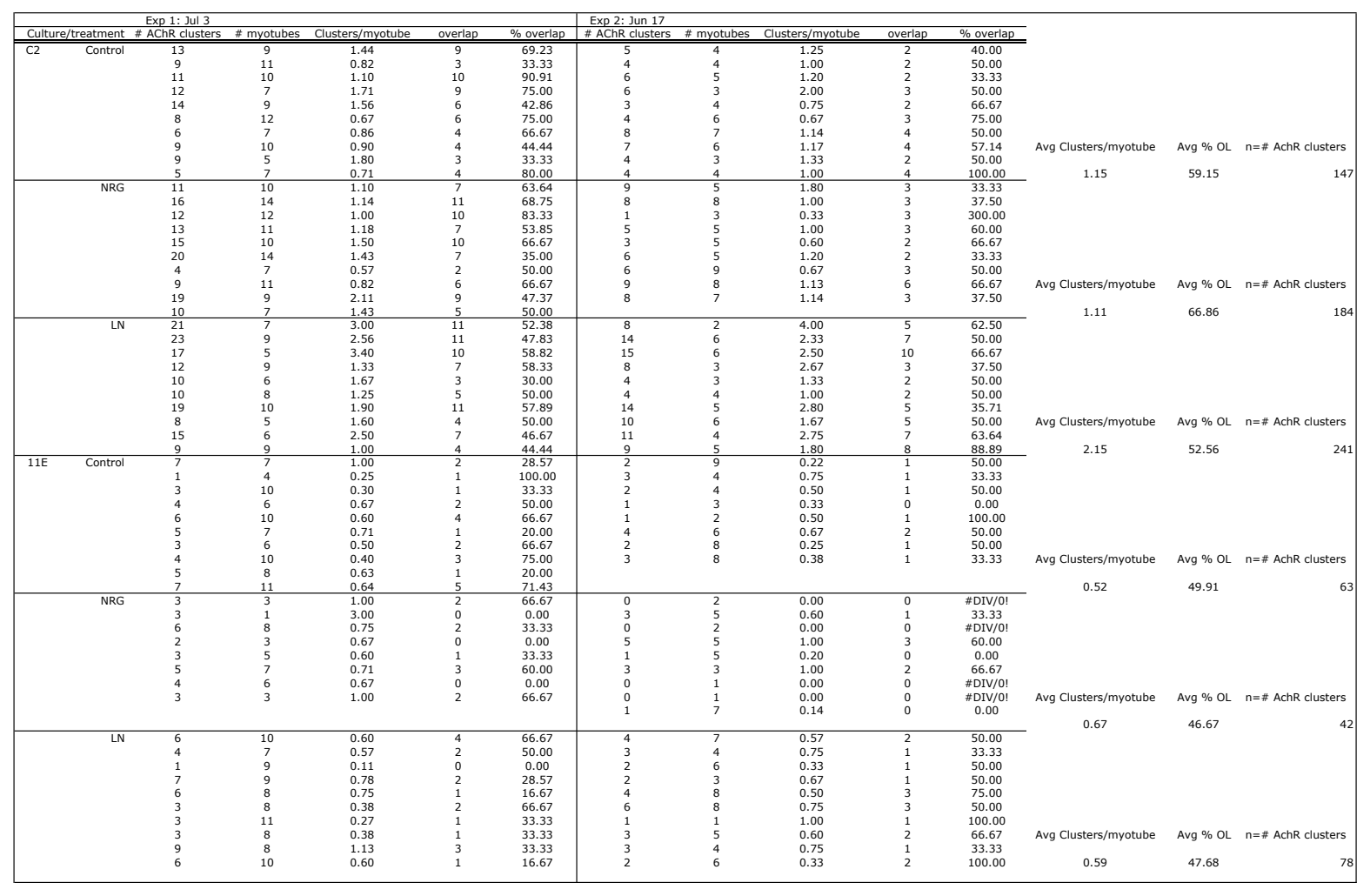




\section{References}

Albert, Y., Whitehead, J., Eldredge, L., Carter, J., Gao, X., and Tourtellotte, W. G. (2005). Transcriptional regulation of myotube fate specification and intrafusal muscle fiber morphogenesis. J Cell Biol 169, 257-268.

Altiok, N., Altiok, S., and Changeux, J. P. (1997). Heregulin-stimulated acetylcholine receptor gene expression in muscle: requirement for MAP kinase and evidence for a parallel inhibitory pathway independent of electrical activity. Embo J 16, 717-725.

Andrechek, E. R., Hardy, W. R., Girgis-Gabardo, A. A., Perry, R. L., Butler, R., Graham, F. L., Kahn, R. C., Rudnicki, M. A., and Muller, W. J. (2002). ErbB2 is required for muscle spindle and myoblast cell survival. Mol Cell Biol 22, 4714-4722.

Apel, E. D., Glass, D. J., Moscoso, L. M., Yancopoulos, G. D., and Sanes, J. R. (1997). Rapsyn is required for MuSK signaling and recruits synaptic components to a MuSKcontaining scaffold. Neuron 18, 623-635.

Apel, E. D., Roberds, S. L., Campbell, K. P., and Merlie, J. P. (1995). Rapsyn may function as a link between the acetylcholine receptor and the agrin-binding dystrophinassociated glycoprotein complex. Neuron 15, 115-126.

Aumailley, M., and Smyth, N. (1998). The role of laminins in basement membrane function. J Anat 193 (Pt 1), 1-21.

Bartoli, M., Ramarao, M. K., and Cohen, J. B. (2001). Interactions of the rapsyn RINGH2 domain with dystroglycan. J Biol Chem 276, 24911-24917.

Bevan, S., and Steinbach, J. H. (1977). The distribution of alpha-bungarotoxin binding sites of mammalian skeletal muscle developing in vivo. J Physiol 267, 195-213.

Bezakova, G., and Ruegg, M. A. (2003). New insights into the roles of agrin. Nat Rev Mol Cell Biol 4, 295-308.

Blau, H. M., Chiu, C. P., and Webster, C. (1983). Cytoplasmic activation of human nuclear genes in stable heterocaryons. Cell 32, 1171-1180.

Borges, L. S., and Ferns, M. (2001). Agrin-induced phosphorylation of the acetylcholine receptor regulates cytoskeletal anchoring and clustering. J Cell Biol 153, 1-12.

Bowe, M. A., Deyst, K. A., Leszyk, J. D., and Fallon, J. R. (1994). Identification and purification of an agrin receptor from Torpedo postsynaptic membranes: a heteromeric complex related to the dystroglycans. Neuron $12,1173-1180$.

Burden, S., and Yarden, Y. (1997). Neuregulins and their receptors: a versatile signaling module in organogenesis and oncogenesis. Neuron 18, 847-855. 
Burgess, R. W., Dickman, D. K., Nunez, L., Glass, D. J., and Sanes, J. R. (2002). Mapping sites responsible for interactions of agrin with neurons. J Neurochem 83, 271284.

Burgess, R. W., Nguyen, Q. T., Son, Y. J., Lichtman, J. W., and Sanes, J. R. (1999). Alternatively spliced isoforms of nerve- and muscle-derived agrin: their roles at the neuromuscular junction. Neuron 23, 33-44.

Burgess, R. W., Skarnes, W. C., and Sanes, J. R. (2000). Agrin isoforms with distinct amino termini: differential expression, localization, and function. J Cell Biol 151, 41-52.

Campanelli, J. T., Roberds, S. L., Campbell, K. P., and Scheller, R. H. (1994). A role for dystrophin-associated glycoproteins and utrophin in agrin-induced AChR clustering. Cell 77, 663-674.

Campbell, K. P. (1995). Three muscular dystrophies: loss of cytoskeleton-extracellular matrix linkage. Cell 80, 675-679.

Carbonetto, S., and Lindenbaum, M. (1995). The basement membrane at the neuromuscular junction: a synaptic mediatrix. Curr Opin Neurobiol 5, 596-605.

Cartaud, A., Coutant, S., Petrucci, T. C., and Cartaud, J. (1998). Evidence for in situ and in vitro association between beta-dystroglycan and the subsynaptic $43 \mathrm{~K}$ rapsyn protein. Consequence for acetylcholine receptor clustering at the synapse. J Biol Chem 273, $11321-11326$.

Cavanagh, P. R., Derr, J. A., Ulbrecht, J. S., Maser, R. E., and Orchard, T. J. (1992). Problems with gait and posture in neuropathic patients with insulin-dependent diabetes mellitus. Diabet Med 9, 469-474.

Cohen, M. W., Jacobson, C., Godfrey, E. W., Campbell, K. P., and Carbonetto, S. (1995). Distribution of alpha-dystroglycan during embryonic nerve-muscle synaptogenesis. J Cell Biol 129, 1093-1101.

Cohen, M. W., Jacobson, C., Yurchenco, P. D., Morris, G. E., and Carbonetto, S. (1997). Laminin-induced clustering of dystroglycan on embryonic muscle cells: comparison with agrin-induced clustering. J Cell Biol 136, 1047-1058.

Cote, P. D., Moukhles, H., Lindenbaum, M., and Carbonetto, S. (1999). Chimaeric mice deficient in dystroglycans develop muscular dystrophy and have disrupted myoneural synapses. Nat Genet 23, 338-342.

DeChiara, T. M., Bowen, D. C., Valenzuela, D. M., Simmons, M. V., Poueymirou, W. T., Thomas, S., Kinetz, E., Compton, D. L., Rojas, E., Park, J. S., et al. (1996). The receptor tyrosine kinase MuSK is required for neuromuscular junction formation in vivo. Cell 85, 501-512. 
Denzer, A. J., Brandenberger, R., Gesemann, M., Chiquet, M., and Ruegg, M. A. (1997). Agrin binds to the nerve-muscle basal lamina via laminin. J Cell Biol 137, 671-683.

Denzer, A. J., Gesemann, M., Schumacher, B., and Ruegg, M. A. (1995). An aminoterminal extension is required for the secretion of chick agrin and its binding to extracellular matrix. J Cell Biol 131, 1547-1560.

Durbeej, M., Henry, M. D., and Campbell, K. P. (1998). Dystroglycan in development and disease. Curr Opin Cell Biol 10, 594-601.

Ernfors, P., Lee, K. F., Kucera, J., and Jaenisch, R. (1994). Lack of neurotrophin-3 leads to deficiencies in the peripheral nervous system and loss of limb proprioceptive afferents. Cell 77, 503-512.

Ervasti, J. M., and Campbell, K. P. (1993). A role for the dystrophin-glycoprotein complex as a transmembrane linker between laminin and actin. J Cell Biol 122, 809-823.

Ervasti, J. M., Ohlendieck, K., Kahl, S. D., Gaver, M. G., and Campbell, K. P. (1990). Deficiency of a glycoprotein component of the dystrophin complex in dystrophic muscle. Nature 345, 315-319.

Ferns, M., Hoch, W., Campanelli, J. T., Rupp, F., Hall, Z. W., and Scheller, R. H. (1992). RNA splicing regulates agrin-mediated acetylcholine receptor clustering activity on cultured myotubes. Neuron 8, 1079-1086.

Ferns, M. J., Campanelli, J. T., Hoch, W., Scheller, R. H., and Hall, Z. (1993). The ability of agrin to cluster AChRs depends on alternative splicing and on cell surface proteoglycans. Neuron 11, 491-502.

Fertuck, H. C., and Salpeter, M. M. (1974). Localization of acetylcholine receptor by 125I-labeled alpha-bungarotoxin binding at mouse motor endplates. Proc Natl Acad Sci U S A 71, 1376-1378.

Fischbach, G. D., and Cohen, S. A. (1973). The distribution of acetylcholine sensitivity over uninnervated and innervated muscle fibers grown in cell culture. Dev Biol 31, 147162.

Fischbach, G. D., and Rosen, K. M. (1997). ARIA: a neuromuscular junction neuregulin. Annu Rev Neurosci 20, 429-458.

Froehner, S. C., Luetje, C. W., Scotland, P. B., and Patrick, J. (1990). The postsynaptic $43 \mathrm{~K}$ protein clusters muscle nicotinic acetylcholine receptors in Xenopus oocytes.

Neuron 5, 403-410.

Fuhrer, C., Gautam, M., Sugiyama, J. E., and Hall, Z. W. (1999). Roles of rapsyn and agrin in interaction of postsynaptic proteins with acetylcholine receptors. J Neurosci 19, 6405-6416. 
Garcia, R. A., Vasudevan, K., and Buonanno, A. (2000). The neuregulin receptor ErbB-4 interacts with PDZ-containing proteins at neuronal synapses. Proc Natl Acad Sci U S A 97, 3596-3601.

Gautam, M., DeChiara, T. M., Glass, D. J., Yancopoulos, G. D., and Sanes, J. R. (1999). Distinct phenotypes of mutant mice lacking agrin, MuSK, or rapsyn. Brain Res Dev Brain Res 114, 171-178.

Gautam, M., Noakes, P. G., Moscoso, L., Rupp, F., Scheller, R. H., Merlie, J. P., and Sanes, J. R. (1996). Defective neuromuscular synaptogenesis in agrin-deficient mutant mice. Cell 85, 525-535.

Gee, S. H., Blacher, R. W., Douville, P. J., Provost, P. R., Yurchenco, P. D., and Carbonetto, S. (1993). Laminin-binding protein 120 from brain is closely related to the dystrophin-associated glycoprotein, dystroglycan, and binds with high affinity to the major heparin binding domain of laminin. J Biol Chem 268, 14972-14980.

Gee, S. H., Montanaro, F., Lindenbaum, M. H., and Carbonetto, S. (1994). Dystroglycanalpha, a dystrophin-associated glycoprotein, is a functional agrin receptor. Cell 77, 675686.

Gesemann, M., Brancaccio, A., Schumacher, B., and Ruegg, M. A. (1998). Agrin is a high-affinity binding protein of dystroglycan in non-muscle tissue. J Biol Chem 273, 600605.

Gesemann, M., Cavalli, V., Denzer, A. J., Brancaccio, A., Schumacher, B., and Ruegg, M. A. (1996). Alternative splicing of agrin alters its binding to heparin, dystroglycan, and the putative agrin receptor. Neuron 16, 755-767.

Glass, D. J., Bowen, D. C., Stitt, T. N., Radziejewski, C., Bruno, J., Ryan, T. E., Gies, D. R., Shah, S., Mattsson, K., Burden, S. J., et al. (1996). Agrin acts via a MuSK receptor complex. Cell 85, 513-523.

Grady, R. M., Zhou, H., Cunningham, J. M., Henry, M. D., Campbell, K. P., and Sanes, J. R. (2000). Maturation and maintenance of the neuromuscular synapse: genetic evidence for roles of the dystrophin--glycoprotein complex. Neuron 25, 279-293.

Guadagno, E., and Moukhles, H. (2004). Laminin-induced aggregation of the inwardly rectifying potassium channel, Kir4.1, and the water-permeable channel, AQP4, via a dystroglycan-containing complex in astrocytes. Glia 47, 138-149.

Henry, M. D., and Campbell, K. P. (1996). Dystroglycan: an extracellular matrix receptor linked to the cytoskeleton. Curr Opin Cell Biol 8, 625-631.

Hippenmeyer, S., Shneider, N. A., Birchmeier, C., Burden, S. J., Jessell, T. M., and Arber, S. (2002). A role for neuregulin1 signaling in muscle spindle differentiation. Neuron 36, 1035-1049. 
Hoch, W., Ferns, M., Campanelli, J. T., Hall, Z. W., and Scheller, R. H. (1993).

Developmental regulation of highly active alternatively spliced forms of agrin. Neuron $11,479-490$.

Hohenester, E., Tisi, D., Talts, J. F., and Timpl, R. (1999). The crystal structure of a laminin G-like module reveals the molecular basis of alpha-dystroglycan binding to laminins, perlecan, and agrin. Mol Cell 4, 783-792.

Holt, K. H., Crosbie, R. H., Venzke, D. P., and Campbell, K. P. (2000). Biosynthesis of dystroglycan: processing of a precursor propeptide. FEBS Lett 468, 79-83.

Hopf, C., and Hoch, W. (1996). Agrin binding to alpha-dystroglycan. Domains of agrin necessary to induce acetylcholine receptor clustering are overlapping but not identical to the alpha-dystroglycan-binding region. J Biol Chem 271, 5231-5236.

Hopf, C., and Hoch, W. (1998). Tyrosine phosphorylation of the muscle-specific kinase is exclusively induced by acetylcholine receptor-aggregating agrin fragments. Eur $\mathrm{J}$ Biochem 253, 382-389.

Hunt, C. C. (1990). Mammalian muscle spindle: peripheral mechanisms. Physiol Rev 70, 643-663.

Ibraghimov-Beskrovnaya, O., Ervasti, J. M., Leveille, C. J., Slaughter, C. A., Sernett, S. W., and Campbell, K. P. (1992). Primary structure of dystrophin-associated glycoproteins linking dystrophin to the extracellular matrix. Nature 355, 696-702.

Jacobson, C., Cote, P. D., Rossi, S. G., Rotundo, R. L., and Carbonetto, S. (2001). The dystroglycan complex is necessary for stabilization of acetylcholine receptor clusters at neuromuscular junctions and formation of the synaptic basement membrane. J Cell Biol $152,435-450$.

Jacobson, C., Duggan, D., and Fischbach, G. (2004). Neuregulin induces the expression of transcription factors and myosin heavy chains typical of muscle spindles in cultured human muscle. Proc Natl Acad Sci U S A 101, 12218-12223.

Jacobson, C., Montanaro, F., Lindenbaum, M., Carbonetto, S., and Ferns, M. (1998). alpha-Dystroglycan functions in acetylcholine receptor aggregation but is not a coreceptor for agrin-MuSK signaling. J Neurosci 18, 6340-6348.

Jones, G., Moore, C., Hashemolhosseini, S., and Brenner, H. R. (1999). Constitutively active MuSK is clustered in the absence of agrin and induces ectopic postsynaptic-like membranes in skeletal muscle fibers. J Neurosci 19, 3376-3383.

Kararizou, E., Manta, P., Kalfakis, N., and Vassilopoulos, D. (2005). Morphometric study of the human muscle spindle. Anal Quant Cytol Histol 27, 1-4. 
Kim, N., Stiegler, A. L., Cameron, T. O., Hallock, P. T., Gomez, A. M., Huang, J. H., Hubbard, S. R., Dustin, M. L., and Burden, S. J. (2008). Lrp4 is a receptor for Agrin and forms a complex with MuSK. Cell 135, 334-342.

Klein, R., Silos-Santiago, I., Smeyne, R. J., Lira, S. A., Brambilla, R., Bryant, S., Zhang, L., Snider, W. D., and Barbacid, M. (1994). Disruption of the neurotrophin-3 receptor gene trkC eliminates la muscle afferents and results in abnormal movements. Nature 368 , 249-251.

Koch, M., Olson, P. F., Albus, A., Jin, W., Hunter, D. D., Brunken, W. J., Burgeson, R. E., and Champliaud, M. F. (1999). Characterization and expression of the laminin gamma3 chain: a novel, non-basement membrane-associated, laminin chain. J Cell Biol $145,605-618$.

Kucera, J., and Walro, J. M. (1992a). Formation of muscle spindles in the absence of motor innervation. Neurosci Lett 145, 47-50.

Kucera, J., and Walro, J. M. (1992b). Superfluousness of motor innervation for the formation of muscle spindles in neonatal rats. Anat Embryol (Berl) 186, 301-309.

Kucera, J., Walro, J. M., and Reichler, J. (1988). Motor and sensory innervation of muscle spindles in the neonatal rat. Anat Embryol (Berl) 177, 427-436.

Kucera, J., Walro, J. M., and Reichler, J. (1993). Differential effects of neonatal denervation on intrafusal muscle fibers in the rat. Anat Embryol (Berl) 187, 397-408.

Lemke, G. (1996). Neuregulins in development. Mol Cell Neurosci 7, 247-262.

Leu, M., Bellmunt, E., Schwander, M., Farinas, I., Brenner, H. R., and Muller, U. (2003). Erbb2 regulates neuromuscular synapse formation and is essential for muscle spindle development. Development 130, 2291-2301.

Lin, W., Burgess, R. W., Dominguez, B., Pfaff, S. L., Sanes, J. R., and Lee, K. F. (2001). Distinct roles of nerve and muscle in postsynaptic differentiation of the neuromuscular synapse. Nature 410, 1057-1064.

Linnoila, J., Wang, Y., Yao, Y., and Wang, Z. Z. (2008). A mammalian homolog of Drosophila tumorous imaginal discs, Tid1, mediates agrin signaling at the neuromuscular junction. Neuron 60, 625-641.

Ma, E., Morgan, R., and Godfrey, E. W. (1994). Distribution of agrin mRNAs in the chick embryo nervous system. J Neurosci 14, 2943-2952.

Ma, E., Morgan, R., and Godfrey, E. W. (1995). Agrin mRNA variants are differentially regulated in developing chick embryo spinal cord and sensory ganglia. J Neurobiol 26, 585-597. 
Ma, L., Huang, Y. Z., Pitcher, G. M., Valtschanoff, J. G., Ma, Y. H., Feng, L. Y., Lu, B., Xiong, W. C., Salter, M. W., Weinberg, R. J., and Mei, L. (2003). Ligand-dependent recruitment of the ErbB4 signaling complex into neuronal lipid rafts. J Neurosci 23, 3164-3175.

McMahan, U. J. (1990). The agrin hypothesis. Cold Spring Harb Symp Quant Biol 55, 407-418.

Meier, T., Hauser, D. M., Chiquet, M., Landmann, L., Ruegg, M. A., and Brenner, H. R. (1997). Neural agrin induces ectopic postsynaptic specializations in innervated muscle fibers. J Neurosci 17, 6534-6544.

Meier, T., Masciulli, F., Moore, C., Schoumacher, F., Eppenberger, U., Denzer, A. J., Jones, G., and Brenner, H. R. (1998). Agrin can mediate acetylcholine receptor gene expression in muscle by aggregation of muscle-derived neuregulins. J Cell Biol 141, 715726.

Michele, D. E., and Campbell, K. P. (2003). Dystrophin-glycoprotein complex: posttranslational processing and dystroglycan function. J Biol Chem 278, 15457-15460.

Montanaro, F., Gee, S. H., Jacobson, C., Lindenbaum, M. H., Froehner, S. C., and Carbonetto, S. (1998). Laminin and alpha-dystroglycan mediate acetylcholine receptor aggregation via a MuSK-independent pathway. J Neurosci 18, 1250-1260.

Montanaro, F., Lindenbaum, M., and Carbonetto, S. (1999). alpha-Dystroglycan is a laminin receptor involved in extracellular matrix assembly on myotubes and muscle cell viability. J Cell Biol 145, 1325-1340.

Moukhles, H., and Carbonetto, S. (2001). Dystroglycan contributes to the formation of multiple dystrophin-like complexes in brain. J Neurochem 78, 824-834.

Muller, K. A., Ryals, J. M., Feldman, E. L., and Wright, D. E. (2008). Abnormal muscle spindle innervation and large-fiber neuropathy in diabetic mice. Diabetes 57, 1693-1701.

Okada, K., Inoue, A., Okada, M., Murata, Y., Kakuta, S., Jigami, T., Kubo, S., Shiraishi, H., Eguchi, K., Motomura, M., et al. (2006). The muscle protein Dok-7 is essential for neuromuscular synaptogenesis. Science 312, 1802-1805.

Phillips, W. D., Maimone, M. M., and Merlie, J. P. (1991). Mutagenesis of the 43-kD postsynaptic protein defines domains involved in plasma membrane targeting and AChR clustering. J Cell Biol 115, 1713-1723.

Reist, N. E., Werle, M. J., and McMahan, U. J. (1992). Agrin released by motor neurons induces the aggregation of acetylcholine receptors at neuromuscular junctions. Neuron 8 , 865-868. 
Richardson, J. K., Sandman, D., and Vela, S. (2001). A focused exercise regimen improves clinical measures of balance in patients with peripheral neuropathy. Arch Phys Med Rehabil 82, 205-209.

Rimer, M., Cohen, I., Lomo, T., Burden, S. J., and McMahan, U. J. (1998). Neuregulins and erbB receptors at neuromuscular junctions and at agrin-induced postsynaptic-like apparatus in skeletal muscle. Mol Cell Neurosci 12, 1-15.

Ruegg, M. A., and Bixby, J. L. (1998). Agrin orchestrates synaptic differentiation at the vertebrate neuromuscular junction. Trends Neurosci 21, 22-27.

Ruegg, M. A., Tsim, K. W., Horton, S. E., Kroger, S., Escher, G., Gensch, E. M., and McMahan, U. J. (1992). The agrin gene codes for a family of basal lamina proteins that differ in function and distribution. Neuron 8, 691-699.

Rupp, F., Payan, D. G., Magill-Solc, C., Cowan, D. M., and Scheller, R. H. (1991). Structure and expression of a rat agrin. Neuron 6, 811-823.

Smalheiser, N. R. (1993). Cranin interacts specifically with the sulfatide-binding domain of laminin. J Neurosci Res 36, 528-538.

Stapley, P. J., Ting, L. H., Hulliger, M., and Macpherson, J. M. (2002). Automatic postural responses are delayed by pyridoxine-induced somatosensory loss. J Neurosci 22, 5803-5807.

Stone, D. M., and Nikolics, K. (1995). Tissue- and age-specific expression patterns of alternatively spliced agrin mRNA transcripts in embryonic rat suggest novel developmental roles. J Neurosci 15, 6767-6778.

Sugiyama, J., Bowen, D. C., and Hall, Z. W. (1994). Dystroglycan binds nerve and muscle agrin. Neuron 13, 103-115.

Sugiyama, J. E., Glass, D. J., Yancopoulos, G. D., and Hall, Z. W. (1997). Laminininduced acetylcholine receptor clustering: an alternative pathway. J Cell Biol 139, 181191.

Swash, M., and Fox, K. P. (1972). The effect of age on human skeletal muscle. Studies of the morphology and innervation of muscle spindles. J Neurol Sci 16, 417-432.

Sweeney, C., Fambrough, D., Huard, C., Diamonti, A. J., Lander, E. S., Cantley, L. C., and Carraway, K. L., 3rd (2001). Growth factor-specific signaling pathway stimulation and gene expression mediated by ErbB receptors. J Biol Chem 276, 22685-22698.

Tansey, M. G., Chu, G. C., and Merlie, J. P. (1996). ARIA/HRG regulates AChR epsilon subunit gene expression at the neuromuscular synapse via activation of phosphatidylinositol 3-kinase and Ras/MAPK pathway. J Cell Biol 134, 465-476. 
Timpl, R., and Brown, J. C. (1996). Supramolecular assembly of basement membranes. Bioessays 18, 123-132.

Tourtellotte, W. G., and Milbrandt, J. (1998). Sensory ataxia and muscle spindle agenesis in mice lacking the transcription factor Egr3. Nat Genet 20, 87-91.

Wallace, B. G., Qu, Z., and Huganir, R. L. (1991). Agrin induces phosphorylation of the nicotinic acetylcholine receptor. Neuron 6, 869-878.

Williamson, R. A., Henry, M. D., Daniels, K. J., Hrstka, R. F., Lee, J. C., Sunada, Y., Ibraghimov-Beskrovnaya, O., and Campbell, K. P. (1997). Dystroglycan is essential for early embryonic development: disruption of Reichert's membrane in Dag1-null mice. Hum Mol Genet 6, 831-841.

Yurchenco, P. D., Cheng, Y. S., and Colognato, H. (1992). Laminin forms an independent network in basement membranes. J Cell Biol 117, 1119-1133.

Zelena, J. (1994). Nerves and Mechanoreceptors - The Role of Innervation in the Development and Maintenance of Mammilian Mechanoreceptors (New York: Chapman and Hall).

Zhang, B., Luo, S., Wang, Q., Suzuki, T., Xiong, W. C., and Mei, L. (2008). LRP4 serves as a coreceptor of agrin. Neuron 60, 285-297.

Zhou, W., and Carpenter, G. (2000). Heregulin-dependent trafficking and cleavage of ErbB-4. J Biol Chem 275, 34737-34743. 